Discussion Papers of the

Max Planck Institute for

Research on Collective Goods

2019/14

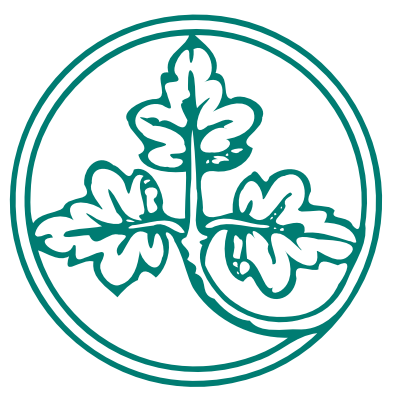

Incomplete-Information

Games in Large Populations with Anonymity

Martin Hellwig 


\title{
Incomplete-Information Games in Large Populations with Anonymity
}

\author{
Martin Hellwig
}

November 2019 


\title{
Incomplete-Information Games in Large Populations with Anonymity*
}

\author{
Martin F. Hellwig \\ Max Planck Institute for Research on Collective Goods \\ Kurt-Schumacher-Str. 10, D - 53113 Bonn, Germany \\ hellwig@coll.mpg.de
}

November 14, 2019

\begin{abstract}
The paper provides mathematical foundations for modeling strategic interdependence with a continuum of agents where uncertainty has an aggregate component and an agent-specific component and the latter satisfies a conditional law of large numbers. This decomposition of uncertainty is implied by a condition of anonymity in beliefs, under which the agent in question considers the other agents' types to be essentially pairwise exchangeable. If there is also anonymity in payoff functions, all strategically relevant aspects of beliefs are contained in an agent's macro beliefs about the cross-section distribution of the other agents' types. The paper also gives conditions under which a function assigning macro beliefs to types is compatible with the existence of a common prior.

Key Words: Incomplete-information games, large populations, belief functions, common priors, exchangeability, conditional independence, conditional exact law of large numbers.
\end{abstract}

JEL: C70, D82, D83.

\footnotetext{
${ }^{*}$ This paper originated in the challenge posed by a referee's asking for the mathematical foundations of the analysis of common priors in Hellwig (2011), now Sections 5.2 and 5.3 of this paper. For helpful discussions and advice, I am grateful to Felix Bierbrauer, Christoph Engel, Alia Gizatulina, Christian Hellwig, and especially Yeneng Sun.
} 


\section{Introduction}

This paper develops mathematical foundations for the study of incompleteinformation games with the following properties:

- The payoff for any one agent depends only on the agent's own characteristics and actions and on the cross-section distribution of actions in the population.

- There are many agents, and each agent considers the effect of his own actions on the cross-section distribution of actions to be negligible.

- Uncertainty can be decomposed into an aggregate component and an agent-specific component, and the latter satisfies an exact law of large numbers.

Such games are not covered by the standard approach to studying strategic interdependence with incomplete information, which considers games with finitely many participants where each participant has beliefs about every other participant's characteristics and actions. ${ }^{1}$

However, in many situations, people do not care about who does what among the other participants. They only care about how many of them engage in any one course of action. This is particularly true for strategic interactions involving large masses of people where aggregate outcomes depend only on aggregate measures of actions. The following examples illustrate the point:

Currency attacks and bank runs: In models of currency attacks and bank runs, the payoff to an agent's choice to attack or to run depends on how many agents are also choosing to attack or to run. Any one agent is therefore concerned about the fraction of people in the population that have received bad signals and are likely to speculate against the currency or run on the bank. ${ }^{2}$

\footnotetext{
${ }^{1}$ This approach was introduced by Harsanyi (1967/8) and Mertens and Zamir (1985).

${ }^{2}$ Whereas the early models of bank runs in Bryant (1980) and Diamond and Dybvig (1983) assumed homogeneous information, since Morris and Shin (1998), the literature on currency attacks and bank runs has aasumed that each agent privately observes a noisy signal of the fundamental. Given the observation of this signal, the agent forms expectations about the value of the fundamental and about the population share of the set of people who will choose to participate in a currency attack or a bank run. If the chances are good that this population share is high enough for the attack to be successful, the agent will also choose to particpate. In addition to Morris and Shin (1998) see C. Hellwig (2002), Rochet and Vives (2004), Goldstein and Pauzner (2005), and Angeletos and Werning (2006).
} 
Insider trading and market microstructure: Strategic behavior in markets with asymmetric information depends on agents' expectations about the relative importance of information trading and liquidity trading. In organized markets in which the identities of traders are not revealed, these expectations concern the distribution of characteristics among the potential traders. $^{3}$

Durable-goods monopoly: A monopolistic seller of a durable commodity does not care about the potential buyers' individual valuations for his good, only about the cross-section distribution of these valuations in the population and the implications of this distribution for the demand that he faces under alternative selling strategies. ${ }^{4}$

Corporate takeover battles: The outcome of a takeover battle for the control of a widely-held corporation depends on the fractions of shareholders that accept the different tender offers, or that reject them. In assessing the alternatives, therefore, each shareholder is concerned with the distribution of actions taken by the other shareholders. ${ }^{5}$

Electoral competition and voting: In voting, the identities of individuals are irrelevant. Only the fractions of the population that vote for or against the given alternatives matter. In models of strategic voting, people form expectations about the distribution of other people's votes. This distribution depends on the distribution of other people's characteristics, i.e., preference parameters or realizations of information variables, and on how these characteritics affect their votes. ${ }^{6}$

Mechanism design and voting for public-good provision: Efficient public-good provision hinges on a comparison of aggregate marginal benefits and the marginal costs of an additional unit of a public good. Aggregate marginal benefits are given by the sum of the marginal benefits of the different participants. People's identities do not matter, only the crosssection distribution of characteristics in the population. In a large economy, this distribution is independent of any one agent's characteristics, and the distribution of reported characteristics is independent of any one agent's report. ${ }^{7}$

In such applications, the notion that any one agent is too insignificant to affect aggregate outcomes is usually formalized by assuming that there

\footnotetext{
${ }^{3}$ See, for example Kyle $(1985,1989)$.

${ }^{4}$ See, e.g., Gul et al. (1985).

${ }^{5}$ See Grossman and Hart (1980) and the subsequent literature.

${ }^{6}$ See, e.g. Lindbeck and Weibull (1987), Alesina and Tabellini (1990).

${ }^{7}$ See Bierbrauer and Hellwig (2010, 2015).
} 
is a continuum of agents. Uncertainty is decomposed into an aggregate component and an agent-specific component, and a law of large numbers is assumed for the latter. The mathematical difficulties inherent in the notion of a continuum of agent-specific random variables are usually ignored.

This is where the present paper steps in. I develop a Harsanyi $(1967 / 68)$ type space framework for studying strategic interdependence when there is a continuum of agents, each one of them insignificant, and each agent has incomplete information about the state of the world. Each agent's characteristics are summarized in terms of an abstract "type". This type determines the agent's probabilistic beliefs about the other agents' types as well as the parameters that enter the agent's payoff function in any strategic game.

Within this formalism, I show that the properties listed above can be derived from two conditions of anonymity, one referring to payoff functions, the other to beliefs. Anonymity in payoff functions postulates that the payoff which an agent expects to obtain from a given action in a strategic game depends only on the agent's own type and action and on the cross-section distribution of the other agents' actions. Which of the other agents is taking which action makes no difference as long as the cross-section distribution of actions is the same.

If all agents choose the same strategy, i.e. the same mapping from types to actions, the cross-section distribution of actions itself determined by the cross-section distribution of types and the common strategy. If different agents choose different strategies, this decomposition is not available, but then the condition of anonymity in beliefs ensures that an agent's expectations about the cross-section distribution of actions is always determined by his expectations about the cross-section distribution of types and the crosssection distribution of strategies. Under this condition, the agent thinks about the other agents' types as essentially pairwise exchangeable random variables.

Exchangeability has very strong consequences. If agent $a$ treats the other agents' types as essentially pairwise exchangeable random variables, a version of De Finetti's Theorem implies that the agent considers the other agents' types to be essentially pairwise conditionally independent and identically distributed. Moreover, if there are many agents, the conditioning variable can be taken to be the distribution of the other agents' types across the population. In this case, the agent's beliefs can be summarized by what I will call his macro beliefs, namely his probabilistic beliefs about the cross-section distribution of types of the other agents. Conditional on this cross-section distribution, the agent considers the other agents' types to be independent and identically distributed with a common conditional proba- 
bility distribution that actually coincides with the cross-section distribution.

With a continuum of agents, the notion of (conditional) independence of agents' types involves a well-known mathematical conundrum. ${ }^{8}$ If there is any agent-specific uncertainty about types at all, independence of the different agents' types implies that, depending on the choice of $\sigma$-algebra on the space of agents, the assignment of types to agents may be nonmeasurable and the notion of "cross-section distribution of types" may not be well defined. Specifically, if the space of agents' names is the Lebesgue unit interval, then, with probability one, the assignment of types to agents is nonmeasurable.

Sun (2006) has proposed to deal with this conundrum by having sufficiently large $\sigma$-algebras on the space of agents and on the product of the space of agents and the underlying probability space so that the random variables of interest are jointly measurable in agents' names and in states of nature and, moreover, integration over both agents' names and states of nature exhibits the Fubini property that the order of integration does not make a difference to the outcome. Subsequent work has refined this approach. ${ }^{9}$ In particular, Qiao et al. (2016) provide a framework for studying a continuum of exchangeable and of conditionally independent random variables. I will follow their approach and rely heavily on their results.

In adapting Sun's approach to games of incomplete information with a continuum of agents, one must bridge the gap between Sun's representation of randomness in terms of a complete probability space and Harsanyi's representation of an agent's beliefs as probability measures over constellations of other agents' types. If one thinks about the belief $b_{a}\left(t_{a}\right)$ of agent $a$ with type $t_{a}$ in isolation, this task is straightforward, though cumbersome. If one thinks about beliefs as a result of conditioning on the observation of one's own type, the task is more difficult, because it is not a priori clear that the condition of completeness of the probability spaces underlying the beliefs $b_{a}\left(t_{a}\right)$ for different types $t_{a}$ can be met for all types simultaneously. I will show that this can be done if the beliefs $b_{a}\left(t_{a}\right)$ of different types $t_{a}$ of agent $a$ are mutually absolutely continuous. I refer to such a belief function as coherent.

The above-mentioned condition of anonymity in beliefs is imposed on the belief $b_{a}\left(t_{a}\right)$ of agent $a$ with type $t_{a}$. It implies that the belief $b_{a}\left(t_{a}\right)$ is fully determined by the agent's macro belief $b_{a}^{*}\left(t_{a}\right)$, i.e. his probabilistic expec-

\footnotetext{
${ }^{8}$ For early accounts, see Judd (1985) and Feldman and Gilles (1986).

${ }^{9}$ See Sun and Zhang (2009), Podczeck (2010), and Qiao et al. (2016). Hammond and Sun $(2003,2008)$ develop a related approach that involves the limits of arbitrarily large finite samples from the given measure space of agents.
} 
tations about the cross-section distribution of the other agents' types, with the proviso that, given this cross-section distribution, the agent considers the other agents' types to be conditionally independent and identically distributed with a conditional probability distribution equal to the cross-section distribution. Together with anonymity in payoff functions, this condition ensures that the agent's expected payoff in a strategic game is fully determined by the agent's macro belief and by the agent's expectations about the crosssection distribution of the other agents' strategies. All strategically relevant features of the belief $b_{a}\left(t_{a}\right)$ are thus contained in the associated macro belief $b_{a}^{*}\left(t_{a}\right)$.

If the belief function is coherent, the condition that $b_{a}\left(t_{a}\right)$ satisfy anonymity in beliefs for all $t_{a}$ is equivalent to the condition that, under any prior that induces the belief function $b_{a}(\cdot)$ as a regular conditional distribution, the types $t_{a^{\prime}}, a^{\prime} \neq a$, of the other agents are essentially pairwise exchangeable. By a version of de Finetti's theorem again, it follows that the agent's prior uncertainty about the other agents' types can also be decomposed into a macro component and a micro component, such that the macro component concerns the cross-section distribution of types and the micro component concerns each agent's individual type, with a conditional probability distribution that coincides with the cross-section distribution of types in the population.

The last part of the paper provides a characterization of common priors and gives conditions for a coherent macro belief function to be compatible with a common prior. Whereas, trivially, every probability distribution over cross-section distributions of types can be used to specify a common prior with associated belief and macro belief functions, not every belief function that satisfies anonymity in beliefs is compatible with the existence of a common prior. A macro belief function that is what I call strongly coherent will be shown to admit a common prior if and only if it satisfies a version of the consistency condition that Harsanyi's (1967/68) gave for the existence of a common prior in a certain two-player game. 


\section{An Incomplete Information Model with a Con- tinuum of Agents}

\subsection{Agents, Types, and Beliefs}

Let $(A, \mathcal{A}, \alpha)$ be a complete atomless measure space of agents with $\alpha(A)=1$. Given this measure space, an incomplete-information model

$$
\left\{T_{a}, \Theta_{a}, \theta_{a}, b_{a}\right\}_{a \in A}
$$

is specified as follows. For each $a \in A, T_{a}$ is a space of abstract "types", and $\Theta_{a}$ is a space of "payoff types", i.e. a space of parameters that may be relevant for the agent's payoffs in any strategic game. Without loss of generality, I assume that the spaces $T_{a}$ and $\Theta_{a}$ are the same for all agents, i.e. that, for some $T$ and $\Theta, T_{a}=T$ and $\Theta_{a}=\Theta$ for all $a \in A$. I also assume that $T$ and $\Theta$ are complete separable metric spaces.

Further, $\theta_{a}$ and $b_{a}$ are mappings such that, for any $t_{a} \in T_{a}, \theta_{a}\left(t_{a}\right) \in$ $\Theta$ determines the agent's payoff function in any strategic game and $b_{a}\left(t_{a}\right)$ specifies the agent's beliefs about other agents' types when his type is $t_{a}$.

The belief $b_{a}\left(t_{a}\right)$ is a probability measure over possible constellations of the other agents' abstract types. The set of such constellations might in principle be identified with the product $T^{A_{-a}}$, where $A_{-a}:=A \backslash\{a\}$ is the set of agents other than $a$. However, this product space is awkward to deal with because, for an arbitrary element of $T^{A_{-a}}$, the assignment of types to agents is not generally measurable and the notion of a cross-section distribution of types is not generally well defined. ${ }^{10}$ Therefore, I assume that the belief type $b_{a}\left(t_{a}\right)$ of agent $a$ with type $t_{a}$ is concentrated on a (very small) subset of possible type constellations.

To specify this subset, I assume that, for some complete probability space $\left(\Omega_{a}\left(t_{a}\right), \mathcal{F}_{a}\left(t_{a}\right), P_{a}\left(t_{a}\right)\right)$ and some measurable function $\tau_{a}\left(\cdot, \cdot \mid t_{a}\right)$ from $\Omega_{a} \times A_{-a}$ to $T$, agent $a$ with abstract type $t_{a}$ assigns probability one to the range $R_{\tau_{a}\left(t_{a}\right)} \subset T^{A_{-a}}$ of the mapping

$$
\left.\omega \rightarrow \boldsymbol{\tau}^{a}\left(\omega \mid t_{a}\right):=\left\{\tau_{a}\left(\omega, a^{\prime} \mid t_{a}\right)\right)\right\}_{a^{\prime} \in A_{-a}} .
$$

If $R_{\tau_{a}\left(t_{a}\right)}$ is endowed with the $\sigma$-algebra $\hat{\mathcal{F}}_{a}\left(t_{a}\right)$ of sets $\hat{F}$ such that for some $F \in \mathcal{F}_{a}\left(t_{a}\right),\left\{t_{a^{\prime}}\right\}_{a^{\prime} \in A_{-a}} \in \hat{F}$ if and only there exists $\omega \in F$ such that $\boldsymbol{\tau}^{a}\left(\omega \mid t_{a}\right)=\left\{t_{a^{\prime}}\right\}_{a^{\prime} \in A_{-a}}$, belief types can be specified as follows.

\footnotetext{
${ }^{10}$ See Judd (1985), Feldman and Gilles (1986).
} 
Belief Types For any $a \in A$ and $t_{a} \in T$, the belief type $b_{a}\left(t_{a}\right)$ is given as

$$
b_{a}\left(t_{a}\right)=P_{a}\left(t_{a}\right) \circ \boldsymbol{\tau}^{a}\left(\cdot \mid t_{a}\right)^{-1}
$$

In the preceding paragraph, I have been deliberately vague about the measurability of the function $\tau_{a}\left(\cdot, \cdot \mid t_{a}\right)$. It might seem natural to require measurability with respect to the usual product $\sigma$-algebra $\mathcal{F}_{a}\left(t_{a}\right) \otimes \mathcal{A}_{-a}$, where $\mathcal{A}_{-a}:=\left\{A^{\prime} \backslash\{a\} \mid A^{\prime} \in \mathcal{A}\right\}$ is the $\sigma$-algebra on $A_{-a}$. . However, as was shown by Sun (2006) and Hammond and Sun (2008), this requirement may preclude any nontrivial uncertainty at the level of individuals. Following Sun (2006) and Qiao et al. (2016), I therefore impose the weaker requirement that $\tau_{a}\left(\cdot, \cdot \mid t_{a}\right)$ be measurable with respect to a rich Fubini extension of $\mathcal{F}_{a}\left(t_{a}\right) \otimes \mathcal{A}_{-a}$, rather than $\mathcal{F}_{a}\left(t_{a}\right) \otimes \mathcal{A}_{-a}$ itself. For this purpose I first introduce the notion of a Fubini extension and then the notion of richness of this extension.

Fubini Extension Given the complete probability spaces $(\Omega, \mathcal{F}, P)$, and $(I, \mathcal{I}, \lambda)$, the probability space $(\Omega \times I, \mathcal{W}, Q)$ is a Fubini extension of the product space $(\Omega \times I, \mathcal{F} \otimes \mathcal{I}, P \otimes \lambda)$ if for any real-valued $Q$ integrable function $f$ on $(\Omega \times I, W)$, (i) the sections $f(\cdot, i)$ and $f(\omega, \cdot)$ are integrable, respectively, on $(\Omega, \mathcal{F}, P)$ for $\lambda$-almost all $i \in I$, and on $(I, \mathcal{I}, \lambda)$ for $P$-almost all $\omega \in \Omega$, and (ii) the functions

$$
i \rightarrow \int_{\Omega} f(\omega, i) d P(\omega) \text { and } \omega \rightarrow \int_{I} f(\omega, i) d \lambda(i)
$$

are integrable, respectively, on $(I, \mathcal{I}, \lambda)$ and $(\Omega, \mathcal{F}, P)$ with

$$
\int_{\Omega \times I} f(\omega, i) d Q=\int_{\Omega}\left[\int_{I} f(\omega, i) d \lambda(i)\right] d P(\omega)=\int_{I}\left[\int_{\Omega} f(\omega, i) d P(\omega)\right] d \lambda(i)
$$

To reflect the fact that the probability space $(\Omega \times I, W, Q)$ has $(\Omega, \mathcal{F}, P)$, and $(I, \mathcal{I}, \lambda)$ as its marginal spaces, as required by the Fubini property, I write $W=\mathcal{F} \otimes \mathcal{I}$ and $Q=P \otimes \lambda$, so the notation

$$
(\Omega \times I, \mathcal{F} \otimes \mathcal{I}, P \otimes \lambda)
$$

indicates that I refer to a Fubini extension of the product $(\Omega \times I, \mathcal{F} \otimes \mathcal{I}$, $P \otimes \lambda)$. 
Richness of the Fubini Extension A Fubini extension $(\Omega \times I, \mathcal{F} \otimes \mathcal{I}, P \otimes$ $\lambda$ ) of a product probability space $(\Omega \times I, \mathcal{F} \otimes \mathcal{I}, P \otimes \lambda)$ is said to be rich if there exists a measurable function $h$ from $(\Omega \times I, \mathcal{F} \otimes \mathcal{I}, P \otimes \lambda)$ to the unit interval such that (i) the random variables $h(\cdot, i), i \in I$, are essentially pairwise independent, i.e., for $\lambda$-almost all $i_{1} \in I$, the random variables $h\left(\cdot, i_{1}\right)$ and $h\left(\cdot, i_{2}\right)$ are independent for $\lambda$-almost all $i_{2} \in I$, and, moreover, (ii) for $\lambda$-almost every $i \in I$, the random variable $h(\cdot, i)$ has a uniform distribution.

The requirement of richness excludes the product space $(\Omega \times I, \mathcal{F} \otimes \mathcal{I}$, $P \otimes \lambda)$. If $h$ is a measurable function from the product space $(\Omega \times I, \mathcal{F} \otimes \mathcal{I}$, $P \otimes \lambda)$ to the unit interval and if the random variables $h(\cdot, i), i \in I$, are essentially pairwise independent, then, as shown in Proposition 2.1 of Sun (2006), the random variables $h(\cdot, i), i \in I$, must be essentially trivial, i.e., for $\lambda$-almost all $i \in I, h(\cdot, i)$ must be a constant random variable, which is not compatible with richness. ${ }^{11}$

Conditions for the existence of a rich Fubini extension are given in Sun (2006), Sun and Zhang (2009), and Podczeck (2010). In particular, Sun (2006) shows that a rich Fubini extension exists if $(I, \mathcal{I}, \lambda)$ is a hyperfinite Loeb space. Sun and Zhang (2009) show that, whereas a rich Fubini extension fails to exist if $I$ is the unit interval with the Lebesgue $\sigma$-algebra, an extended Lebesgue unit interval, with a larger $\sigma$-algebra, does permit the construction of a rich Fubini extension of the product $(\Omega \times I, \mathcal{F} \otimes \mathcal{I}, P \otimes \lambda)$.

In the present context, I set $(I, \mathcal{I}, \lambda)=\left(A_{-a}, \mathcal{A}_{-a}, \alpha_{-a}\right)$, where $\alpha_{-a}:=$ $\alpha \mid \mathcal{A}_{-a}$ is the restriction of the measure $\alpha$ to $\mathcal{A}_{-a} \subset \mathcal{A}$, and assume that the function $\tau_{a}\left(\cdot, \cdot \mid t_{a}\right)$ in the specification of agent $a$ 's beliefs is measurable with respect to a rich Fubini extension $\mathcal{F}_{a}\left(t_{a}\right) \otimes \mathcal{A}_{-a}$ of the product $\mathcal{F}_{a}\left(t_{a}\right) \otimes \mathcal{A}_{-a}$.

\subsection{Strategic Games}

Given an incomplete-information model $\left\{T, \Theta, \theta_{a}, b_{a}\right\}_{a \in A}$, a strategic game of incomplete information is defined by specifying, for each $a \in A$, an action set $S_{a}$ and a payoff function $u_{a}: \Theta \times \prod_{a^{\prime} \in A} S_{a} \rightarrow \mathbb{R}$. I assume that the actions sets $S_{a}$ are the same for all agents, i.e. that, for some $S, S_{a}=S$ for all $a \in A$, so the domain of the payoff function $u_{a}$ can be written as $\Theta \times S^{A}$. I also assume that $S$ is a compact metric space.

\footnotetext{
${ }^{11}$ Proposition 4 in Hammond and Sun (2008) provides a version of this result with essential pairwise conditional independence.
} 
A strategy for agent $a$ is a function $\sigma_{a}: T \rightarrow S$ that indicates, for each $t_{a} \in T$, the action the agent is to take if his type is $t_{a}$. The strategy that agent $a$ chooses will as a rule depend on the agent's expectations about the other agents' strategy choices. If $\sigma_{a}\left(\cdot, a^{\prime}\right)$ is the strategy that agent $a$ expects to be followed by agent $a^{\prime} \in A_{-a}$, the expected payoff of agent $a$ with abstract type $t_{a}$ from choosing an action $s_{a}$ is given as

$$
\int_{R_{\tau}} u_{a}\left(\theta_{a}\left(t_{a}\right), s_{a},\left\{\sigma_{a}\left(t_{a^{\prime}}, a^{\prime}\right)\right\}_{a^{\prime} \in A_{-a}}\right) d b_{a}\left(\left\{t_{a^{\prime}}\right\}_{a^{\prime} \in A_{-a}} \mid t_{a}\right) .
$$

A strategy $s_{a}^{*}$ of agent $a$ is a best response to the strategies $\sigma_{a}\left(\cdot, a^{\prime}\right)$ for $a^{\prime} \in A_{-a}$ that the agent expects the other agents to choose if

$$
\begin{aligned}
& \int_{R_{\tau}} u_{a}\left(\theta_{a}\left(t_{a}\right), s_{a}^{*}\left(t_{a}\right),\left\{\sigma_{a}\left(t_{a^{\prime}}, a^{\prime}\right)\right\}_{a^{\prime} \in A_{-a}}\right) d b_{a}\left(\left\{t_{a^{\prime}}\right\}_{a^{\prime} \in A_{-a}} \mid t_{a}\right) \\
\geq & \int_{R_{\tau}} u_{a}\left(\theta_{a}\left(t_{a}\right), s_{a},\left\{\sigma_{a}\left(t_{a^{\prime}}, a^{\prime}\right)\right\}_{a^{\prime} \in A_{-a}}\right) d b_{a}\left(\left\{t_{a^{\prime}}\right\}_{a^{\prime} \in A_{-a}} \mid t_{a}\right)
\end{aligned}
$$

for all $t_{a} \in T$ and all $s_{a} \in S$. In this best-response condition, the comparison between the agent's payoff expectation under the choice $s_{a}^{*}\left(t_{a}\right)$ and an alternative choice $s_{a}$ depends on the agent's expectations about the constellation $\left\{\sigma_{a}\left(t_{a^{\prime}}, a^{\prime}\right)\right\}_{a^{\prime} \in A_{-a}}$ of the other agents' actions as well as the agent's own payoff type $\theta_{a}\left(t_{a}\right)$. The agent's expectations about the constellation $\left\{\sigma_{a}\left(t_{a^{\prime}}, a^{\prime}\right)\right\}_{a^{\prime} \in A_{-a}}$ of the other agents' actions in turn depends on the agents' expectaions about the constellation $\left\{t_{a^{\prime}}\right\}_{a^{\prime} \infty A_{-a}}$ and the constellation $\left\{\sigma_{a}\left(\cdot, a^{\prime}\right)\right\}_{a^{\prime} \infty A_{-a}}$ of strategies that the agent expects the other agents to follow. ${ }^{12}$

In this very general formulation, the other agents' names may matter. It may make a difference to agent $a$ whether a given action $s$ is taken by agent $a^{\prime}$ or by agent $a^{\prime \prime}$; in forming his expectations, the agent may also make a difference between the events $t_{a^{\prime}}=t$ and the $t_{a^{\prime \prime}}=t$, for any $t$. To eliminate this possibility, I will introduce two conditions of anonymity under which the agent does not care whether a given action or a given type pertains to agent $a^{\prime}$ or to agent $a^{\prime \prime}$, one condition on payoff functions and one condition on belief functions.

\footnotetext{
${ }^{12}$ This dependence is the reason why I formulate beliefs in terms of probability measures over constellations $\left\{t_{a^{\prime}}\right\}_{a^{\prime} \in A_{-a}}$, rather than pairs $\left(a^{\prime}, t_{a^{\prime}}\right)$ like Sun (2006) or Qiao et al. (2016). In Sun (2006) and Qiao et al. (2016), the simpler approach works because they only consider applications in which the economic variables that are of interest, such as aggregate resource requirements, take the form $\int f\left(a, t_{a}\right) d \alpha(a)$. This functional form, however, does not allow for the more general kinds of strategic interdependence that appear in many games.
} 


\section{Anonymity}

\subsection{Anonymity in Payoff Functions}

It seems most natural to define anonymity by the condition that payoffs are unchanged under any measurable permutation of the other agents' names. If the measure space $\left(A_{-a}, \mathcal{A}_{-a}, \alpha_{-a}\right)$ is homogeneous, for example, if $\left(A_{-a}, \mathcal{A}_{-a}, \alpha_{-a}\right)$ is a hyperfinite Loeb space, this definition of anonymity is equivalent to the requirement that other agents' actions affect the payoff

$$
u_{a}\left(\theta_{a}\left(t_{a}\right), s_{a},\left\{\sigma_{a}\left(t_{a^{\prime}}, a^{\prime}\right)\right\}_{a^{\prime} \in A_{-a}}\right)
$$

of agent $a$ only through their cross-section distribution $D\left(\left\{\sigma_{a}\left(t_{a^{\prime}}, a^{\prime}\right)\right\}_{a^{\prime} \in A_{-a}}\right)$, an element of the space $\mathcal{M}(S)$ of probability measures on $S .{ }^{13}$ Because the latter requirement is more convenient to work with, I define:

Anonymity in Payoff Functions For any $a \in A$, there exists a function $u_{a}^{*}: \Theta \times S \times \mathcal{M}(S) \rightarrow \mathbb{R}$ such that, for all $t_{a} \in T$ and all $s_{a} \in S$,

$$
u_{a}\left(\theta_{a}\left(t_{a}\right), s_{a},\left\{\sigma_{a}\left(t_{a^{\prime}}, a^{\prime}\right)\right\}_{a^{\prime} \in A_{-a}}\right)=u_{a}^{*}\left(\theta_{a}\left(t_{a}\right), s_{a}, D\left(\left\{\sigma_{a}\left(t_{a^{\prime}}, a^{\prime}\right)\right\}_{a^{\prime} \in A_{-a}}\right)\right)
$$

for all constellations of actions $\left\{\sigma_{a}\left(t_{a^{\prime}}, a^{\prime}\right)\right\}_{a^{\prime} \in A_{-a}}$ for which the crosssection distribution $D\left(\left\{\sigma_{a}\left(t_{a^{\prime}}, a^{\prime}\right)\right)\right.$ is well defined.

Remark 3.1 If the map

$$
\left(a^{\prime}, t_{a^{\prime}}\right) \rightarrow \sigma_{a}\left(t_{a^{\prime}}, a^{\prime}\right)
$$

from $A_{-a} \times T$ to $S$ is measurable, then, for any $t_{a} \in T$, the cross-section distribution of actions $D\left(\left\{\sigma_{a}\left(t_{a^{\prime}}, a^{\prime}\right)\right\}_{a^{\prime} \in A_{-a}}\right)$ is $b_{a}\left(t_{a}\right)$-almost surely well defined.

Proof. By assumption, $b_{a}\left(t_{a}\right)$ is concentrated on the range $R_{\tau_{a}\left(t_{a}\right)}$ of the function $\tau^{a}\left(\cdot \mid t_{a}\right)$. Therefore it suffices to show that, for every $\omega \in \Omega$, the cross-section distribution $D\left(\left\{\sigma_{a}\left(\tau_{a}\left(\omega, a^{\prime} \mid t_{a}\right), a^{\prime}\right)\right\}_{a^{\prime} \in A_{-a}}\right)$ is well defined.

\footnotetext{
${ }^{13}$ See Khan and Sun (1999), Section 4. Homogeneity fails to hold if $(A, \mathcal{A}, \alpha)$ is the Lebesgue unit interval. In this case, the requirement that the strategy constellation $\left\{\sigma_{a}\left(t_{a^{\prime}}, a^{\prime}\right)\right\}_{a^{\prime} \in A_{-a}}$ affects $u_{a}$ only through the distribution $D\left(\left\{\sigma_{a}\left(t_{a^{\prime}}, a^{\prime}\right)\right\}_{a^{\prime} \in A_{-a}}\right)$ is stronger than the requirement of invariance under measurable permutations of names. Because the Lebesgue $\sigma$-algebra is based on neighbourhood structures, the set of measurable permutations of names is too small for equivalence of the two notions of anonymity.
} 
Because the function $\tau^{a}\left(\cdot \mid t_{a}\right)$ from $\Omega_{a}\left(t_{a}\right) \times A_{-a}$ to $T$ is measurable with respect to the Fubini $\sigma$-algebra $\mathcal{F}_{a}\left(t_{a}\right) \otimes \mathcal{A}_{-a}$, the assumption that (3.2) is measurable implies that, for given $t_{a}$, the map

$$
\left(\omega, a^{\prime}\right) \rightarrow \varphi^{a}\left(\omega, a^{\prime} \mid t_{a}\right):=\sigma_{a}\left(\tau_{a}\left(\omega, a^{\prime} \mid t_{a}\right), a^{\prime}\right)
$$

from $\Omega_{a}\left(t_{a}\right) \times A_{-a}$ to $S$ is also measurable with respect to the Fubini $\sigma$ algebra $\mathcal{F}_{a}\left(t_{a}\right) \otimes \mathcal{A}_{-a}$. For every $\omega \in \Omega_{a}\left(t_{a}\right)$, therefore, the section $\varphi^{a}\left(\omega, \cdot \mid t_{a}\right)$ of the function $\varphi^{a}\left(\cdot, \cdot \mid t_{a}\right)$ is measurable with respect to $\mathcal{A}_{-a}$, and one may write

$$
D\left(\left\{\sigma_{a^{\prime}}^{a}\left(\tau_{a}\left(\omega, a^{\prime}\right)\right\}_{a^{\prime} \in A_{-a}}\right)=\alpha_{-a} \circ\left(\varphi^{a}\left(\omega, \cdot \mid t_{a}\right)\right)^{-1},\right.
$$

i.e., $D\left(\left\{\sigma_{a}\left(\tau_{a}\left(\omega, a^{\prime} \mid t_{a}\right), a^{\prime}\right)\right\}_{a^{\prime} \in A_{-a}}\right)$ is well defined.

With anonymity in payoff functions, the expected payoff (2.6) takes the form

$$
\int_{R_{\tau}} u_{a}^{*}\left(\theta_{a}\left(t_{a}\right), s_{a}, D\left(\left\{\sigma_{a}\left(t_{a^{\prime}}, a^{\prime}\right)\right\}_{a^{\prime} \in A_{-a}}\right)\right) d b_{a}\left(\left\{t_{a^{\prime}}\right\}_{a^{\prime} \in A_{-a}} \mid t_{a}\right) .
$$

The cross-section distribution of actions, $D\left(\left\{\sigma_{a}\left(t_{a^{\prime}}, a^{\prime}\right)\right\}_{a^{\prime} \in A_{-a}}\right)$, depends on the interplay between the constellations $\left\{\sigma_{a^{\prime}}^{a}(\cdot)\right\}_{a^{\prime} \in A_{-a}}$ and $\left\{t_{a^{\prime}}\right\}_{a^{\prime} \in A_{-a}}$ of strategies and of types. If the agent expects that all other agents use the same (measurable) strategy $\sigma_{a}\left(\cdot, a^{\prime}\right)=\sigma_{a}: T \rightarrow S$, this interplay takes a very simple form and we obtain

$$
D\left(\left\{\sigma_{a}\left(t_{a^{\prime}}, a^{\prime}\right)\right\}_{a^{\prime} \in A_{-a}}\right)=D\left(\left\{t_{a^{\prime}}\right\}_{a^{\prime} \in A_{-a}}\right) \circ\left(\sigma_{a}\right)^{-1},
$$

where $D\left(\left\{t_{a^{\prime}}\right\}_{a^{\prime} \in A_{-a}}\right)$ is the cross-section distribution of types. In this case, the agent is only concerned about the distribution of the other agents' types and does not care about which agent has which type.

However, the assumption that agent $a$ expects all other agents to use the same strategy is problematic. After all, the other agents' strategy choices are endogenous. With enough symmetry assumptions on the exogenous data, namely the functions $u_{a^{\prime}}^{*}, \theta_{a^{\prime}}, b_{a^{\prime}}$, in equilibrium, their strategy choices may in fact be symmetric, but that is a very special case. ${ }^{14}$ I will therefore follow an alternative approach and impose an additional anonymity condition on beliefs.

\footnotetext{
${ }^{14}$ Even then, it might be the case that a Bayes-Nash equilibrium involves a symmetric set of asymmetric strategy choice, rather than a single strategy that is chosen by all.
} 


\subsection{Anonymity in Beliefs}

The fundamental idea is that, in forming his beliefs, agent $a$ treats the other agents symmetrically in the sense that the joint distribution of the types of any two of them is unaffected if their names are interchanged.

Anonymity in Beliefs For any $a \in A$ and $t_{a} \in T$, the measure $b_{a}\left(t_{a}\right)$ satisfies anonymity in beliefs if, under this measure, the types $t_{a^{\prime}}$ of agents $a^{\prime} \neq a$ are essentially pairwise exchangeable, i.e., there exists a probability measure $p_{a}\left(\cdot \mid t_{a}\right)$ on $T^{2}$ such that, for $\alpha_{-a^{-}}$-almost all $a^{1} \in A_{-a}$, one has

$$
b_{a}\left(\left\{t_{a^{1}} \in B_{1}\right\} \cap\left\{t_{a^{2}} \in B_{2}\right\} \mid t_{a}\right)=p_{a}\left(B_{1} \times B_{2} \mid t_{a}\right)=p_{a}\left(B_{2} \times B_{1} \mid t_{a}\right)
$$

for $\alpha_{-a}$-almost all $a^{2} \in A_{-a}$ and all Borel sets $B_{1}, B_{2} \subset T$.

Anonymity in beliefs corresponds to de Finetti's notion of exchangeability. For sequences of random variables, de Finetti's Theorem asserts the equivalence of exchangeability with the property of conditional independence relative to some underlying $\sigma$-algebra. Kingman (1978) provides a lucid account of the argument and shows that the conditioning $\sigma$-algebra may be identified with the algebra generated by the limiting sample distribution of the process; moreover, by a conditional law of large numbers, this limiting sample distribution of the process coincides with the conditional probability distribution of any one of the random variables given the conditioning $\sigma$-algebra.

For models with a continuum of random variables, Hammond and Sun (2003, 2008) have shown that the property of essential pairwise exchangeability is equivalent to the property of essential pairwise conditional independence relative to some countably generated $\sigma$-algebra, with identical conditional distributions. Relying on the framework of a Fubini extension, Qiao et al. (2016) have shown that the conditioning $\sigma$-algebra may be identified with the algebra generated by the cross-section distributions of the random variables in question; moreover, by a conditional law of large numbers, the conditional probability distribution of any one of the random variables and the cross-section sample distribution coincide.

In the following, I use these results to explore the implications of anonymity in beliefs for incomplete-information games with a continuum of agents. A key role is played by the cross-section type distribution $D\left(\left\{t_{a^{\prime}}\right\}_{a^{\prime} \in A_{-a}}\right)$. This distribution is an element of the space $\mathcal{M}(T)$ of probability measures on $T$. 
Upon endowing this space with the topology of weak convergence and the associated Borel $\sigma$-algebra, one obtains:

Remark 3.2 For any $t_{a} \in T$, the map $\left\{t_{a^{\prime}}\right\}_{a^{\prime} \in A_{-a}} \rightarrow D\left(\left\{t_{a^{\prime}}\right\}_{a^{\prime} \in A_{-a}}\right)$ from type constellations in $R_{\tau_{a}\left(t_{a}\right)}$ to cross-section distributions of types, i.e. measures in $\mathcal{M}(T)$, is measurable.

Proof. Given the definition of $R_{\tau_{a}\left(t_{a}\right)}$ and the specification of the $\sigma$-algebra $\hat{\mathcal{F}}_{a}\left(t_{a}\right)$ on $R_{\tau_{a}\left(t_{a}\right)}$, it suffices to show that the map

$$
\omega \rightarrow \delta(\omega):=D\left(\left\{\tau_{a}\left(\omega, a^{\prime} \mid t_{a}\right)\right\}_{a^{\prime} \in A_{-a}}\right)
$$

from $\Omega_{a}\left(t_{a}\right)$ to $\mathcal{M}(T)$ is measurable. Since $T$ is a complete separable metrice space and $\mathcal{M}(T)$ has the topology of weak convergence, there exists a sequence $\left\{g_{i}\right\}$ of bounded continuous functions from $T$ to the unit interval such that the mapping

$$
\Delta \rightarrow\left\{\int g_{i}(t) d \Delta(t)\right\}_{i=1}^{\infty}
$$

defines a homeomorphism between $\mathcal{M}(T)$ and a subset of $\mathbb{R}^{\infty}$ (Parthasarathy, 1967, p. 47). To prove the lemma, it therefore suffices so show that, for any $i$, the mapping

$$
\omega \rightarrow \int g_{i}(t) d \delta(t \mid \omega)
$$

from $\Omega$ to $\mathbb{R}$ is measurable. The definition of $\delta$ implies that, for any $\omega$,

$$
\delta(\omega)=\alpha_{-a} \circ\left(\tau_{a}\left(\omega, \cdot \mid t_{a}\right)\right)^{-1},
$$

and therefore,

$$
\int g_{i}(t) d \delta(t \mid \omega)=\int g_{i}\left(\tau_{a}\left(\omega, a^{\prime} \mid t_{a}\right)\right) d \alpha_{-a}\left(a^{\prime}\right)
$$

Measurability of (3.7) is therefore implied by the assumption that $\tau_{a}\left(\cdot, \cdot \mid t_{a}\right)$ is measurable with respect to a Fubini extension.

The following proposition adapts Proposition 3 of Qiao et al. (2016) to the present setting.

Proposition 3.3 Let $\hat{\mathcal{D}} \subset \hat{\mathcal{F}}_{a}\left(t_{a}\right)$ be the $\sigma$-algebra on $R_{\tau_{a}\left(t_{a}\right)}$ that is generated by the mapping

$$
\left\{t_{a^{\prime}}\right\}_{a^{\prime} \in A_{-a}} \rightarrow D\left(\left\{t_{a^{\prime}}\right\}_{a^{\prime} \in A_{-a}}\right) .
$$


Anonymity in beliefs is equivalent to the requirement that, for any $t_{a} \in$ $T$, under the measure $b_{a}\left(t_{a}\right)$, conditionally on $\hat{\mathcal{D}}$, the types $t_{a^{\prime}}, a^{\prime} \in A_{-a}$, are essentially pairwise independent and identically distributed, i.e., that for $\alpha_{-a}$-almost all $a^{1} \in A_{-a}$, the agent considers the types $t_{a^{1}}$ and $t_{a^{2}}$ to be conditionally independent given $\hat{\mathcal{D}}$, for $\alpha_{-a^{-}}$almost all $a^{2} \in A_{-a}$.

Moreover, the sample distribution $D\left(\left\{t_{a^{\prime}}\right\}_{a^{\prime} \in A_{-a}}\right)$ of types in the population is $b_{a}\left(t_{a}\right)$-almost surely equal to the common conditional distribution of types $t_{a^{1}}$ in the population, i.e., for $\alpha_{-a^{-}}$almost all $a^{1} \in A_{-a}$, the mapping (3.9) from $R_{\tau_{a}\left(t_{a}\right)}$ to $\mathcal{M}(T)$ is a regular conditional distribution for $t_{a^{1}}$ given $\hat{\mathcal{D}}$.

Proof. Given the definition of $b_{a}\left(t_{a}\right)$, anonymity in beliefs is equivalent to the condition that the random variables $\tau_{a}\left(\cdot, a^{\prime} \mid t_{a}\right), a^{\prime} \in A_{-a}$, are essentially pairwise exchangeable. Moreover, $\hat{\mathcal{D}}=\left\{\boldsymbol{\tau}^{a}\left(F \mid t_{a}\right) \mid F \in \mathcal{D}\right\}$, where $\mathcal{D}$ is the sub- $\sigma$-algebra of $\mathcal{F}_{a}\left(t_{a}\right)$ that is generated by the mapping (3.6). The proposition is therefore equivalent to the statement that, with anonymity in beliefs, conditionally on $\mathcal{D}$, the random variables $\tau_{a}\left(\cdot, a^{\prime} \mid t_{a}\right), a^{\prime} \in A_{-a}$, are essentially pairwise independent and identically distributed with regular conditional probability distribution $\delta(\cdot)$. This latter statement is implied by Proposition 3 of Qiao et al. (2016).

Proposition 3.3 has two components. One component asserts the equivalence of anonymity in beliefs with essential pairwise conditional independence (with identical conditional distributions). The other component asserts a conditional law of large numbers, namely under the belief $b_{a}\left(t_{a}\right)$, the cross-section distribution of types is almost surely equal to the common conditional distribution of the other agents' types given the $\sigma$-algebra $\hat{\mathcal{D}}$, which in turn implies that the sample distributions can be interpreted as conditional probability distributions.

\subsection{Expected Payoffs under Anonymity in Beliefs}

Proposition 3.3 provides the basis for the following result about the relation between the cross-section distributions of actions and of types.

Proposition 3.4 If the map $\left(a^{\prime}, t_{a^{\prime}}\right) \rightarrow \sigma_{a^{\prime}}^{a}\left(t_{a^{\prime}}\right)$ from $A_{-a} \times T$ to $S$ is measurable, then, under the assumption of anonymity in beliefs,

$$
D\left(\left\{\sigma_{a}\left(t_{a^{\prime}}, a^{\prime}\right)\right\}_{a^{\prime} \in A_{-a}}\right)=\int_{A_{-a}} D\left(\left\{t_{a^{\prime}}\right\}_{a^{\prime} \in A_{-a}}\right) \circ \sigma_{a}\left(\cdot, a^{\prime}\right)^{-1} d \alpha_{-a}\left(a^{\prime}\right),
$$

$b_{a}\left(t_{a}\right)$-almost surely. 
Proof. Since $b_{a}\left(t_{a}\right)$ is concentrated on the range $R_{\tau_{a}\left(t_{a}\right)}$ of the function $\boldsymbol{\tau}^{a}\left(\cdot \mid t_{a}\right)$, it suffices to show that the equation

$$
D\left(\left\{\sigma_{a}\left(\tau_{a}\left(\omega, a^{\prime} \mid t_{a}\right), a^{\prime}\right)\right\}_{a^{\prime} \in A_{-a}}\right)=\int_{A_{-a}} \delta(\omega) \circ \sigma_{a}\left(\cdot, a^{\prime}\right)^{-1} d \alpha_{-a}\left(a^{\prime}\right)
$$

holds for $P_{a}\left(t_{a}\right)$-almost all $\omega \in \Omega_{a}\left(t_{a}\right)$, where, for any $\omega, \delta(\omega) \in \mathcal{M}(T)$ is again given by (3.6). Since $S$ is a complete separable metric space and $\mathcal{M}(S)$ has the topology of weak convergence, there exists a sequence $\left\{h_{i}\right\}$ of continuous functions from $S$ to the unit interval such that the mapping

$$
\Delta \rightarrow\left\{\int_{S} h_{i}(s) d \Delta(s)\right\}_{i=1}^{\infty}
$$

defines a homeomorphism between $\mathcal{M}(S)$ and a subset of $\mathbb{R}^{\infty}$ (Parthasarathy, 1967, p. 47). Thus it suffices to prove that, for $P_{a}\left(t_{a}\right)$-almost all $\omega \in \Omega_{a}\left(t_{a}\right)$, the equation

$$
\int_{A_{, a}} h_{i}\left(\sigma_{a}\left(\tau_{a}\left(\omega, a^{\prime} \mid t_{a}\right), a^{\prime}\right)\right) d \alpha_{-a}\left(a^{\prime}\right)=\int_{A_{-a}} \int_{T} h_{i}\left(\sigma_{a}\left(t, a^{\prime}\right)\right) d \delta(t \mid \omega) d \alpha_{-a}\left(a^{\prime}\right)
$$

holds for all $i$. For any $i$, consider the mapping

$$
\left(\omega, a^{\prime}\right) \rightarrow \eta_{i}\left(\omega, a^{\prime} \mid t_{a}\right):=h_{i}\left(\sigma_{a}\left(\tau_{a}\left(\omega, a^{\prime} \mid t_{a}\right), a^{\prime}\right)\right.
$$

from $\Omega_{a}\left(t_{a}\right) \times A_{-a}$ to the unit interval, and note that the left-hand side of (3.12) is equal to the integral $\int_{A_{a}} \eta_{i}\left(\omega, a^{\prime} \mid t_{a}\right) d \alpha_{-a}\left(a^{\prime}\right)$. Because the map $\left(a^{\prime}, t_{a^{\prime}}\right) \rightarrow \sigma_{a}\left(t_{a^{\prime}}, a^{\prime}\right)$ from $A_{-a} \times T$ to $S$ is measurable, the map $\left(\omega, a^{\prime}\right) \rightarrow$ $\eta_{i}\left(\omega, a^{\prime} \mid t_{a}\right)$ is measurable with respect to the Fubini extension $\mathcal{F}_{a}\left(t_{a}\right) \otimes \mathcal{A}_{-a}$ of the product $\sigma$-algebra $\mathcal{F}_{a}\left(t_{a}\right) \otimes \mathcal{A}_{-a}$.

By the argument in the proof of Proposition 3.3, anonymity in beliefs implies that, conditionally on the $\sigma$-algebra $\mathcal{D}$ that is generated by the mapping (3.6), the random variables $\eta_{i}\left(\cdot, a^{\prime} \mid t_{a}\right)$ are essentially pairwise independent. By Corollary 2 in Qiao et al. (2016), it follows that

$$
\int_{A_{. a}} \eta_{i}\left(\cdot, a^{\prime} \mid t_{a}\right) d \alpha_{-a}\left(a^{\prime}\right)=\int_{A_{-a}} E\left[\eta_{i}\left(\cdot, a^{\prime} \mid t_{a}\right) \mid \mathcal{D}\right] d \alpha_{-a}\left(a^{\prime}\right)
$$

$P_{a}\left(t_{a}\right)$-almost surely. The argument in the proof of Proposition 3.3 also

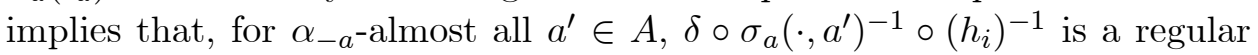
conditional distribution for $\eta_{i}\left(\cdot, a^{\prime} \mid t_{a}\right)$ given $\mathcal{D}$. For $\alpha_{-a^{-a l m o s t}}$ all $a^{\prime} \in A$, therefore,

$$
E\left[\eta_{i}\left(\cdot, a^{\prime} \mid t_{a}\right) \mid \delta(\omega)=\cdot\right]=\int h_{i}\left(\sigma_{a}\left(t, a^{\prime}\right)\right) d \Delta(t \mid)
$$


for all $\Delta \in \mathcal{M}(T), P_{a}\left(t_{a}\right)$-almost surely. From (3.13) - (3.15), it follows that, for any $i$,

$$
\begin{aligned}
\int_{A_{. a}} h_{i}\left(\sigma_{a}\left(\tau_{a}\left(\omega, a^{\prime} \mid t_{a}\right), a^{\prime}\right)\right) d \alpha_{-a}\left(a^{\prime}\right) & =\int_{A_{A a}} \eta_{i}\left(\omega, a^{\prime} \mid t_{a}\right) d \alpha_{-a}\left(a^{\prime}\right) \\
& =\int_{A_{-a}} E\left[\eta_{i}\left(\cdot, a^{\prime} \mid t_{a}\right) \mid \delta(\omega)\right] d \alpha_{-a}\left(a^{\prime}\right) \\
& =\int_{A_{-a}} \int_{T} h_{i}\left(\sigma_{a}\left(t, a^{\prime}\right)\right) d \delta(t \mid \omega) d \alpha_{-a}\left(a^{\prime}\right)
\end{aligned}
$$

for all $\omega$ outside a $P_{a}\left(t_{a}\right)$-null set $N_{i}$. Therefore (3.12) holds for all $i$ and all $\omega$ outside the union $\cup_{i} N_{i}$. Because a countable union of null sets is still a null set, it follows that (3.12) holds for all $i, P_{a}\left(t_{a}\right)$-almost surely.

Thus, with anonymity in beliefs, the cross-section distribution of actions of agents other than $a$ can be written as a function of the constellation $\left\{\sigma_{a}\left(\cdot, a^{\prime}\right)\right\}_{a^{\prime} \in A_{-a}}$ of strategies of the other agents and the cross-section distribution $D\left(\left\{t_{a^{\prime}}\right\}_{a^{\prime} \in A_{-a}}\right)$ of types of the other agents. By inspection of (3.10), one sees that, in fact, the strategy constellation $\left\{\sigma_{a}\left(\cdot, a^{\prime}\right)\right\}_{a^{\prime} \in A_{-a}}$ enters only through the cross-section distribution

$$
D\left(\left\{\sigma_{a}\left(\cdot, a^{\prime}\right)\right\}_{a^{\prime} \in A_{-a}}\right):=\alpha_{-a} \circ\left(\boldsymbol{\sigma}^{a}\right)^{-1},
$$

where $\boldsymbol{\sigma}^{a}$ is the mapping that assigns to each agent $a^{\prime} \in A_{-a}$ the strategy $\sigma_{a}\left(\cdot, a^{\prime}\right)$ that agent $a$ expects agent $a^{\prime}$ to follow. Equation (3.10) can thus be rewritten in the form

$$
\begin{aligned}
D\left(\left\{\sigma_{a^{\prime}}^{a}\left(t_{a^{\prime}}\right)\right\}_{a^{\prime} \in A_{-a}}\right) & =\int_{A_{-a}} D\left(\left\{t_{a^{\prime}}\right\}_{a^{\prime} \in A_{-a}}\right) \circ s(\cdot)^{-1} d\left(\alpha_{-a} \circ\left(\boldsymbol{\sigma}^{a}\right)^{-1}\right) \\
& =\int_{A_{-a}} D\left(\left\{t_{a^{\prime}}\right\}_{a^{\prime} \in A_{-a}}\right) \circ s(\cdot)^{-1} d \Sigma^{a}(s)
\end{aligned}
$$

where

$$
\Sigma^{a}:=\alpha_{-a} \circ\left(\boldsymbol{\sigma}^{a}\right)^{-1}
$$

denotes the distribution of other agents' strategies that is anticipated by agent $a$.

With anonymity in beliefs as well as payoffs, the expected payoff of agent $a$ with abstract type $t_{a}$ from choosing an action $s_{a}$ can therefore be written as 


$$
\begin{aligned}
& \int_{R_{\tau}} u_{a}\left(\theta_{a}\left(t_{a}\right), s_{a},\left\{\sigma_{a}\left(t_{a^{\prime}}, a^{\prime}\right)\right\}_{a^{\prime} \in A_{-a}}\right) d b_{a}\left(\left\{t_{a^{\prime}}\right\}_{a^{\prime} \in A_{-a}} \mid t_{a}\right) \\
= & \int_{R_{\tau}} u_{a}^{*}\left(\theta_{a}\left(t_{a}\right), s_{a}, D\left(\left\{\sigma_{a}\left(t_{a^{\prime}}, a^{\prime}\right)\right\}_{a^{\prime} \in A_{-a}}\right)\right) d b_{a}\left(\left\{t_{a^{\prime}}\right\}_{a^{\prime} \in A_{-a}} \mid t_{a}\right) \\
= & \int_{R_{\tau}} u_{a}^{*}\left(\theta_{a}\left(t_{a}\right), s_{a}, \int_{A_{-a}} D\left(\left\{t_{a^{\prime}}\right\}_{a^{\prime} \in A_{-a}}\right) \circ s(\cdot)^{-1} d \Sigma^{a}(s)\right) d b_{a}\left(\left\{t_{a^{\prime}}\right\}_{a^{\prime} \in A_{-a}} \mid t_{a}\right) .
\end{aligned}
$$

\subsection{Expected Payoffs and Macro Beliefs}

In (3.17), the agent's belief $b_{a}\left(t_{a}\right)$ matters only to the extent that it concerns the cross-section type distribution $D\left(\left\{t_{a^{\prime}}\right\}_{a^{\prime} \in A_{-a}}\right)$. It is therefore convenient to replace the formulation of beliefs in terms of type constellations by one in terms of type distributions. I will refer to such beliefs over type distributions as macro beliefs.

Macro Beliefs For any $a \in A$ and $t_{a} \in T$, the macro belief of agent $a$ with belief type $b_{a}\left(t_{a}\right)$ is a probability measure $b_{a}^{*}\left(t_{a}\right)$ over the space $\mathcal{M}(T)$ of cross-section distributions of abstract types that is defined by the equation

$$
b_{a}^{*}\left(t_{a}\right)=b_{a}\left(t_{a}\right) \circ D(\cdot)^{-1} .
$$

From (3.17), one now obtains the following corollary to Proposition 3.4:

Corollary 3.5 With anonymity in payoff functions and in beliefs, the expected payoff of agent a with abstract type $t_{a}$ from choosing an action $s_{a}$ takes the form

$$
\int_{R_{\tau}} u_{a}^{*}\left(\theta_{a}\left(t_{a}\right), s_{a}, \int_{A_{-a}} \Delta \circ s(\cdot)^{-1} d \Sigma^{a}(s) d b_{a}^{*}\left(\Delta \mid t_{a}\right),\right.
$$

A strategy $s_{a}^{*}$ of agent $a$ is a best response to the constellation $\boldsymbol{\sigma}^{a}=\left\{\sigma_{a}\left(\cdot, a^{\prime}\right)\right\}_{a^{\prime} \in A_{-a}}$ that the agent expects the other agents to choose if and only if the inequality

$$
\begin{aligned}
& \int_{R_{\tau}} u_{a}^{*}\left(\theta_{a}\left(t_{a}\right), s_{a}^{*}\left(t_{a}\right), \int_{A_{-a}} \Delta \circ s(\cdot)^{-1} d \Sigma^{a}(s) d b_{a}^{*}\left(\Delta \mid t_{a}\right)\right. \\
& \geq \int_{R_{\tau}} u_{a}^{*}\left(\theta_{a}\left(t_{a}\right), s_{a}, \int_{A_{-a}} \Delta \circ s(\cdot)^{-1} d \Sigma^{a}(s) d b_{a}^{*}\left(\Delta \mid t_{a}\right)\right.
\end{aligned}
$$

holds for all $t_{a} \in T$ and all $s_{a} \in S$. 
Anonymity in payoff functions and anonymity in beliefs jointly can thus be used to transform the incomplete-information model (2.1) in which agents form beliefs and expectations as to what are the types and actions of every single other agent, into a model in which agents only form beliefs and expectations about cross-section distribution of types and cross-section distributions of strategies of the other agents. Whereas the other agents' "names" play a substantive role in the best-response condition (2.7), they do not even appear in the best-response condition (3.20).

The key to this simplification is provided by Propositions 3.3 and 3.4, showing that anonymity in beliefs permits a simple decomposition of an agent's probabilistic beliefs into a macro component and a micro component, his beliefs about the cross-section distribution of types and his beliefs about each individual's type, conditional on the cross-section distribution. The micro component of the agent's beliefs fully determined by the fact that, conditional on the cross-section type distribution, the other agents' types are viewed as essentially pairwise independent and identically distributed, with a conditional probability distribution that is equal to the cross-section distribution.

\subsection{The Scope of Macro Uncertainty}

I conclude this section with a result showing that, if the Fubini extension $\mathcal{F}_{a}\left(t_{a}\right) \otimes \mathcal{A}_{-a}$ is rich, then under anonymity in beliefs, the macro belief of agent $a$ with type $t_{a}$ can be any measure on $\mathcal{M}(T)$, i.e., there is no restriction on macro beliefs. The only restriction on admissible beliefs comes from the principle that, for a given cross-section distribution of types, the conditional probability distribution of the random variable $\tau_{a}\left(\cdot, a^{\prime}\right)$ that represents the type of agent $a^{\prime} \neq a$ is equal to the cross-section distribution of types, i.e. that the agent's beliefs about the other agents' types respect the conditional law of large numbers that is implied by exchangeability.

Proposition 3.6 Let $\beta$ be any probability measure on $\mathcal{M}(T)$. If the Fubini extension $\left(\Omega_{a}\left(t_{a}\right) \times A_{-a}, \mathcal{F}_{a}\left(t_{a}\right) \otimes \mathcal{A}_{-a}, P_{a}\left(t_{a}\right) \otimes \alpha_{-a}\right)$ is rich, there exists an $\mathcal{F}_{a}\left(t_{a}\right) \otimes \mathcal{A}_{-a}$-measurable mapping $\tau_{a}\left(\cdot \mid t_{a}\right)$ from $\Omega_{a}\left(t_{a}\right) \times A_{-a}$ to $T$ such that the belief $b_{a}\left(t_{a}\right)$ that is given by (2.3) satisfies anonymity in beliefs and the associated macro belief is $b_{a}^{*}\left(t_{a}\right)=\beta$.

Proof. The proof proceeds along similar lines as the proof of Proposition 5.3 in Sun (2006). Let $\beta \in \mathcal{M}(\mathcal{M}(T))$ by given. By standard arguments, 
there exists an $\mathcal{M}(T)$-valued random variable $\tilde{\delta}_{\beta}$ on $\left(\Omega_{a}\left(t_{a}\right), \mathcal{F}_{a}\left(t_{a}\right), P_{a}\left(t_{a}\right)\right)$ such that the probability distribution of $\tilde{\delta}_{\beta}$ is $\beta$, i.e., $P_{a}\left(t_{a}\right) \circ \tilde{\delta}_{\beta}^{-1}=\beta$.

By Lemma A.5 of Sun (2006), there also exists a measurable function $f$ from $\mathcal{M}(T) \times[0,1]$ into $T$ such that for any $\delta \in \mathcal{M}(T)$,

$$
\ell \circ f(\delta, \cdot)^{-1}=\delta
$$

where $\ell$ is the uniform distribution on $[0,1]$. Given this function $f$ and the random variable $\tilde{\delta}_{\beta}$, define a mapping $\tau_{a}\left(\cdot, \cdot \mid t_{a}\right)$ from $\Omega_{a}\left(t_{a}\right) \times A_{-a}$ into $T$ such that, for any $\omega \in \Omega_{a}\left(t_{a}\right)$ and $a^{\prime} \in A_{-a}$,

$$
\tau_{a}\left(\omega, a^{\prime} \mid t_{a}\right)=f\left(\tilde{\delta}_{\beta}(\omega), h\left(\omega, a^{\prime}\right)\right)
$$

where $h$ is the function given by the richness of the Fubini extension $\left(\Omega_{a}\left(t_{a}\right) \times\right.$ $\left.A_{-a}, \mathcal{F}_{a}\left(t_{a}\right) \otimes \mathcal{A}_{-a}, P_{a}\left(t_{a}\right) \otimes \alpha_{-a}\right)$.

The function $\tau_{a}(\cdot, \cdot)$ is measurable with respect to the $\sigma$-algebra $\mathcal{F}_{a}\left(t_{a}\right) \otimes$ $\mathcal{A}_{-a}$. In fact, $\tau_{a}\left(\cdot, \cdot \mid t_{a}\right)$ is the composition of the measurable function $f$ : $\mathcal{M}(T) \times[0,1] \rightarrow T$ with the function $H: \Omega \times A_{-a} \rightarrow \mathcal{M}(T) \times[0,1]$ that is given by setting

$$
H\left(\omega, a^{\prime}\right)=\left(\tilde{\delta}_{\beta}(\omega), h\left(\omega, a^{\prime}\right)\right)
$$

for any $\omega \in \Omega_{a}\left(t_{a}\right)$ and $a^{\prime} \in A_{-a}$. Because the map $\omega \rightarrow \tilde{\delta}_{\beta}(\omega)$ is measurable with respect to $\mathcal{F}$ and the map $\left(\omega, a^{\prime}\right) \rightarrow h\left(\omega, a^{\prime}\right)$ is measurable with respect to $\mathcal{F} \otimes \mathcal{A}_{-a}$, the map $\left(\omega, a^{\prime}\right) \rightarrow H\left(\omega, a^{\prime} \mid t_{a}\right)$ is measurable with respect to $\mathcal{F} \otimes \mathcal{A}_{-a}$, and so is the map $\left(\omega, a^{\prime}\right) \rightarrow \tau_{a}\left(\omega, a^{\prime} \mid t_{a}\right)=f\left(H\left(\omega, a^{\prime}\right)\right)$.

Let $\mathcal{C}$ be the sub- $\sigma$-algebra of $\mathcal{F}$ that is induced by $\tilde{\delta}$. Because $T$ is a complete separable metric space, $\mathcal{M}(T)$ is also a complete separable metric space, and $\mathcal{C}$ is countably generated. Because the random variables $h\left(\cdot, a^{\prime}\right)$, $a^{\prime} \in A_{-a}$, are essentially pairwise independent, Proposition 3 in Hammond and Sun (2006) implies that they are also essentially pairwise conditionally independent given $\mathcal{C}$. As in Remark 1 of Hammond and Sun (2008), it follows that the random pairs $\left(\tilde{\delta}_{\beta}(\cdot), h\left(\cdot, a^{\prime}\right)\right), a^{\prime} \in A_{-a}$, are also essentially pairwise conditionally independent given $\mathcal{C}$ and so are the random variables $\tau_{a}\left(\cdot, a^{\prime} \mid t_{a}\right)=f\left(\tilde{\delta}_{\beta}(\cdot), h\left(\cdot, a^{\prime}\right)\right), a^{\prime} \in A_{-a}$. By Proposition 3.3 above, it follows that the belief $b_{a}\left(t_{a}\right)$ that is given by (2.3) satisfies anonymity in beliefs.

Moreover, because, for $\alpha_{-a^{-}}$-almost every $a^{\prime} \in A_{-a}$, the random variable $h\left(\cdot, a^{\prime}\right)$ has the uniform distribution $\ell,(3.21)$ and (3.22) imply that, for $\alpha_{-a^{-}}$ almost every $a^{\prime} \in A_{-a}$, conditional on the event $\tilde{\delta}_{\beta}(\cdot)=\delta$, the probability distribution of $\tau_{a}\left(\cdot, a^{\prime} \mid t_{a}\right)$ is almost surely equal to $\delta$. For $\alpha_{-a}$-almost every $a^{\prime} \in A_{-a}$, therefore the function $\tilde{\delta}_{\beta}(\cdot)$ is a regular conditional distribution for $\tau_{a}\left(\cdot, a^{\prime} \mid t_{a}\right)$ given the $\sigma$-algebra $\mathcal{C}_{\tilde{\delta}_{\beta}}$ that is generated by $\tilde{\delta}_{\beta}$. By Corollary 
2 of Qiao et al. (2016) and the fact that, conditionally on $\mathcal{C}_{\tilde{\delta}_{\beta}}$, the random variables $\tau_{a}\left(\cdot, a^{\prime} \mid t_{a}\right), a^{\prime} \in A_{-a}$, are essentially pairwise independent, one also infers that

$$
D\left(\left\{\tau_{a}\left(\omega, a^{\prime} \mid t_{a}\right)\right\}_{a^{\prime} \in A_{-a}}\right)=\tilde{\delta}_{\beta}(\omega),
$$

$P_{a}\left(t_{a}\right)$-almost surely, and, therefore, that the macro belief $b_{a}^{*}\left(t_{a}\right)$ must coincide with the probability distribution $\beta$ of the random variable $\tilde{\delta}_{\beta}$.

Except for purposes of notation, the construction in the proof of Proposition 3.6 makes no reference to the type $t_{a}$ of agent $a$. The belief $b_{a}\left(t_{a}\right)=$ $P_{a}\left(t_{a}\right) \circ \boldsymbol{\tau}^{a}\left(\cdot \mid t_{a}\right)^{-1}$ that results from this construction is fully determined by the indicated macro belief $\beta$. Moreover, it depends on $\beta$ only through the random variable $\tilde{\delta}_{\beta}$. The following remark states this observation formally.

Remark 3.7 The belief $b_{a}\left(t_{a}\right)$ in Proposition 3.6 takes the form $b_{a}\left(t_{a}\right)=$ $\int_{\mathcal{M}(T)} \hat{b}_{a}(\delta) d \beta(\delta)$, where $\hat{b}(\cdot)$ is a mapping from $\mathcal{M}(T)$ to the space of beliefs.

\section{Coherent Belief Functions, Agent-Specific Pri- ors, and Anonymity in Beliefs}

\subsection{Coherence of Belief Functions}

The game theoretic literature contains two disctinct interpretations of the belief $b_{a}\left(t_{a}\right)$ of agent $a$ with type $t_{a}$. In one interpretation, this belief is given as one of the exogenous data of the model. For any $a \in A, t_{a} \in T$, the belief $b_{a}\left(t_{a}\right)$ was taken as given, without any account of how the probability spaces $\left(\Omega_{a}\left(t_{a}\right), \mathcal{F}_{a}\left(t_{a}\right), P_{a}\left(t_{a}\right)\right)$ and mappings $\tau_{a}\left(\cdot, \cdot \mid t_{a}\right)$ for different $t_{a}$ and different $a$ relate to each other.

In another interpretation, the belief $b_{a}\left(t_{a}\right)$ of agent $a$ with type $t_{a}$ is interpreted as the result of agent $a$ 's observing some information and adapting his beliefs accordingly. In this approach, the type $t_{a}$ of agent $a$ is seen as the realization of a random variable $\tilde{t}_{a}$, and the belief $b_{a}\left(t_{a}\right)$ is the result of conditioning on the event $\tilde{t}_{a}=t_{a}$.

With this interpretation of belief functions, the question of how the probability spaces $\left(\Omega_{a}\left(t_{a}\right), \mathcal{F}_{a}\left(t_{a}\right), P_{a}\left(t_{a}\right)\right)$ and mappings $\tau_{a}\left(\cdot, \cdot \mid t_{a}\right)$ for different $t_{a}$ and different $a$ relate to each other cannot be avoided. Two distinct questions arise. First, for any given agent $a$, under what conditions can the beliefs $b_{a}\left(t_{a}\right), t_{a} \in T$, be interpreted as resulting from the agent's conditioning on the observation of $t_{a}$ ? Second, if the beliefs $b_{a}\left(t_{a}\right), t_{a} \in T$, of all agents 
$a \in A$ can be interpreted as results of conditioning, under what conditions can the underlying prior be taken to be the same for all agents, i.e. under what conditions do the given belief functions admit a common prior? I will address these two questions in turn, the first one in the remainder of this section, the second one in Section 5 below.

If the belief function $b_{a}(\cdot)$ of agent $a$ is to be interpreted as a regular conditional distribution, there must be some underlying probability space that is independent of $t_{a}$. The question is whether such a space can be specified in a way that is compatible with the assumption that, for each $t_{a} \in$ $T$, the probability measure $b_{a}\left(t_{a}\right)$ is the distribution of a random variable $\boldsymbol{\tau}^{a}\left(\cdot \mid t_{a}\right)=\left\{\tau_{a}\left(\cdot, a^{\prime} \mid t_{a}\right)\right\}_{a^{\prime} \in A_{-a}}$ that is defined on some complete probability space $\left(\Omega_{a}\left(t_{a}\right), \mathcal{F}_{a}\left(t_{a}\right), P_{a}\left(t_{a}\right)\right)$ where $\tau_{a}\left(\cdot, \cdot \mid t_{a}\right)$ is measurable with respect to a rich Fubini extension of the product $\mathcal{F}_{a}\left(t_{a}\right) \times \mathcal{A}_{-a}$.

The answer to this question turns out to be positive if the belief function satisfies an additional condition of coherence, which ensures that the beliefs $b_{a}\left(t_{a}\right)$ that are associated with different types $t_{a} \in T$ all have the same domain and the same null sets.

Coherence of Belief Functions The belief function $b_{a}(\cdot)$ is said to be coherent if, for any $t_{a}$ and $\hat{t}_{a}$ in $T$, the probability measures $b_{a}\left(t_{a}\right)$ and $b_{a}\left(\hat{t}_{a}\right)$ are mutually absolutely continuous, i.e., any set $F \in T^{A_{-a}}$ satisfies $F \in \hat{\mathcal{F}}_{a}\left(t_{a}\right)$ and $b_{a}\left(F \mid t_{a}\right)>0$ if and only if it also satisfies $F \in \hat{\mathcal{F}}_{a}\left(\hat{t}_{a}\right)$ and $b_{a}\left(F \mid \hat{t}_{a}\right)>0$.

Lemma 4.1 If the belief function $b_{a}(\cdot)$ is coherent, the ranges $R_{\boldsymbol{\tau}^{a}\left(t_{A}\right)}$ of the mappings $\tau_{a}\left(\cdot, \cdot \mid t_{a}\right)$ and the $\sigma$-algebras $\hat{\mathcal{F}}_{a}\left(t_{a}\right)$ are the same for all $t_{a} \in T$, i.e. there exists a measurable space $\left(R_{a}, \hat{\mathcal{F}}_{a}\right)$ such that for all $t_{a} \in T, R_{\boldsymbol{\tau}^{a}\left(t_{A}\right)}=$ $R_{a} \subset T^{A_{-a}}$ and $\hat{\mathcal{F}}_{a}\left(t_{a}\right)=\hat{\mathcal{F}}_{a}$.

Proof. Fix some $\hat{t}_{a} \in T$. I claim that, for any $t_{a} \in T$ and any $F \in \hat{\mathcal{F}}_{a}\left(t_{a}\right)$, it must be the case that $F \in \hat{\mathcal{F}}_{a}\left(\hat{t}_{a}\right)$. If $b_{a}\left(F \mid t_{a}\right)>0$, the claim follows directly from the assumption that the belief function is coherent. If $b_{a}\left(F \mid t_{a}\right)=0$, the claim follows by observing that $R_{\boldsymbol{\tau}^{a}\left(t_{A}\right)} \backslash F \in \hat{\mathcal{F}}_{a}\left(t_{a}\right)$ and $b_{a}\left(R_{\boldsymbol{\tau}^{a}\left(t_{A}\right)} \backslash F \mid t_{a}\right)=$ 1 , so by the coherence of the belief function $b_{a}(\cdot), R_{\tau^{a}\left(t_{A}\right)} \backslash F \in \hat{\mathcal{F}}_{a}\left(\hat{t}_{a}\right)$ and therefore $F \in \hat{\mathcal{F}}_{a}\left(\hat{t}_{a}\right)$. Thus $\hat{\mathcal{F}}_{a}\left(t_{a}\right) \subset \hat{\mathcal{F}}_{a}\left(\hat{t}_{a}\right)$.

Since $R_{\boldsymbol{\tau}^{a}\left(t_{A}\right)} \in \hat{\mathcal{F}}_{a}\left(t_{a}\right)$, for any $t_{a} \subset T$, it follows that $R_{\boldsymbol{\tau}^{a}\left(t_{A}\right)} \in \hat{\mathcal{F}}_{a}\left(\hat{t}_{a}\right)$ and therefore, that $R_{\boldsymbol{\tau}^{a}\left(t_{A}\right)} \subset R_{\boldsymbol{\tau}^{a}\left(\hat{t}_{A}\right)}$. 
By a completely symmetric argument, it must also be the case that, for any $t_{a} \in T, \hat{\mathcal{F}}_{a}\left(\hat{t}_{a}\right) \subset \hat{\mathcal{F}}_{a}\left(t_{a}\right)$ and $R_{\boldsymbol{\tau}^{a}\left(\hat{t}_{A}\right)} \subset R_{\boldsymbol{\tau}^{a}\left(t_{A}\right)}$. The lemma follows immediately.

Proposition 4.2 If the belief function $b_{a}(\cdot)$ is coherent, the measurable spaces $\left(\Omega_{a}\left(t_{a}\right), \mathcal{F}_{a}\left(t_{a}\right)\right)$ and the mappings $\tau_{a}\left(\cdot, \cdot \mid t_{a}\right)$ can be taken to be the same for all $t_{a}$, i.e., for some fixed measurable space $\left(\Omega_{a}, \overline{\mathcal{F}}_{a}\right)$ and some fixed mapping $\tau_{a}: \Omega_{a} \times A_{-a} \rightarrow T$, there exist probability measures $\bar{P}_{a}\left(t_{a}\right), t_{a} \in T$, such that (i) for every $t_{a} \in T$, the probability space $\left(\Omega_{a}, \overline{\mathcal{F}}_{a}, \bar{P}_{a}\left(t_{a}\right)\right)$ is complete, (ii), for every $t_{a} \in T, b_{a}\left(t_{a}\right)=\bar{P}_{a}\left(t_{a}\right) \circ\left(\boldsymbol{\tau}^{a}\right)^{-1}$, where, for any $\omega \in \Omega_{a}$, $\boldsymbol{\tau}^{a}(\omega):=\left\{\tau_{a}\left(\omega, a^{\prime}\right)\right\}_{a^{\prime} \in A_{-a}}$, and (iii) the mapping $\tau_{a}$ is measurable with respect to a Fubini extension of the product $\sigma$-algebra $\overline{\mathcal{F}}_{a} \otimes \mathcal{A}_{-a}$.

Proof. Fix some $\hat{t}_{a} \in T$ and set $\Omega_{a}=\Omega_{a}\left(\hat{t}_{a}\right)$ and $\tau_{a}(\cdot, \cdot)=\tau_{a}\left(\cdot, \cdot \mid \hat{t}_{a}\right)$. Given that the range $R_{\boldsymbol{\tau}^{a}\left(\hat{t}_{a}\right)}$ is endowed with the $\sigma$-algebra $\hat{\mathcal{F}}_{a}\left(\hat{t}_{a}\right)$, let $\mathcal{F}_{a} \subset$ $\mathcal{F}_{a}\left(t_{a}\right)$ be the coarsest $\sigma$-algebra on $\Omega_{a}$ with respect to which the function $\omega \rightarrow \boldsymbol{\tau}^{a}(\omega)=\left\{\tau_{a}\left(\omega, a^{\prime}\right)\right\}_{a^{\prime} \in A_{-a}}$ is measurable. Thus, $\mathcal{F}_{a}$ consists of the sets $F$ taking the form

$$
F=\left\{\omega \in \Omega_{a} \mid \boldsymbol{\tau}^{a}(\omega) \in \hat{F}(F)\right\}
$$

for some set $\hat{F}(F) \in \hat{\mathcal{F}}_{a}\left(\hat{t}_{a}\right)$.

By Lemma 4.1, the coherence of the belief function $b_{a}(\cdot)$ implies that, for any $t_{a} \in T$ and any $F \in \mathcal{F}_{a}$, the set $\hat{F}(F)$ belongs to the $\sigma$-algebra $\hat{\mathcal{F}}_{a}\left(t_{a}\right)$ so, under the measure $b_{a}\left(t_{a}\right)$, the probability $b_{a}\left(\hat{F}(F) \mid t_{a}\right)$ is well defined. For any $t_{a} \in T$, one may therefore define a probability measure $\hat{P}_{a}\left(t_{a}\right)$ on $\left(\Omega_{a}, \mathcal{F}_{a}\right)$ by setting

$$
\hat{P}_{a}\left(F \mid t_{a}\right)=b_{a}\left(\hat{F}(F) \mid t_{a}\right) .
$$

Given $\hat{P}_{a}\left(t_{a}\right)$ and $\left(\Omega_{a}, \mathcal{F}_{a}\right)$, I further define $\overline{\mathcal{F}}_{a}\left(t_{a}\right)$ and $\bar{P}_{a}\left(t_{a}\right)$ so that $\left(\Omega_{a}, \overline{\mathcal{F}}_{a}\left(t_{a}\right), \bar{P}_{a}\left(t_{a}\right)\right)$ is the completion of $\left(\Omega_{a}, \mathcal{F}_{a}, \hat{P}_{a}\left(t_{a}\right)\right)$, i.e. $\overline{\mathcal{F}}_{a}\left(t_{a}\right)$ is the smallest $\sigma$-algebra on $\Omega_{a}$ that contains all subsets of $\hat{P}_{a}\left(t_{a}\right)$-null sets in $\mathcal{F}_{a}$, and $\bar{P}_{a}\left(t_{a}\right)$ is the unique extension of $\hat{P}_{a}\left(t_{a}\right)$ from $\mathcal{F}_{a}$ to $\overline{\mathcal{F}}_{a}\left(t_{a}\right)$.

By the coherence of the belief function $b_{a}(\cdot)$, the $\hat{P}_{a}\left(t_{a}\right)$-null sets in $\mathcal{F}_{a}$ are the same for all $t_{a} \in T$. Therefore the class of subsets of $\hat{P}_{a}\left(t_{a}\right)$-null sets in $\mathcal{F}_{a}$ is the same for all $t_{a} \in T$. Therefore, the $\sigma$-algebra $\overline{\mathcal{F}}_{a}\left(t_{a}\right)$ that is generated by $\mathcal{F}_{a}$ and the class of subsets of $\hat{P}_{a}\left(t_{a}\right)$-null sets in $\mathcal{F}_{a}$ is the same for all $t_{a} \in T$ and can be written as $\overline{\mathcal{F}}_{a}$.

By construction, for any $t_{a} \in T$, the probability space $\left(\Omega_{a}, \overline{\mathcal{F}}_{a}, \bar{P}_{a}\left(t_{a}\right)\right)$ is complete and $b_{a}\left(t_{a}\right)=\bar{P}_{a}\left(t_{a}\right) \circ\left(\boldsymbol{\tau}^{a}\right)^{-1}$, i.e. $\left(\Omega_{a}, \overline{\mathcal{F}}_{a}, \bar{P}_{a}\left(t_{a}\right)\right)$ has properties (i) and (ii) in the statement of the proposition. 
To prove that $\left(\Omega_{a}, \overline{\mathcal{F}}_{a}, \bar{P}_{a}\left(t_{a}\right)\right)$ also has property (iii), let $\mathcal{F}_{a}\left(\hat{t}_{a}\right) \otimes \mathcal{A}_{-a}$ be the Fubini extension of $\mathcal{F}_{a}\left(\hat{t}_{a}\right) \otimes \mathcal{A}_{-a}$ with respect to which the function $\tau_{a}(\cdot, \cdot)=\tau_{a}\left(\cdot, \cdot \mid \hat{t}_{a}\right)$ is measurable. Let $\mathcal{W}$ be the class of sets $B \in \mathcal{F}_{a}\left(\hat{t}_{a}\right) \otimes$ $\mathcal{A}_{-a}$ for which the sections $B_{a^{\prime}}=\left\{\omega \in \Omega_{a} \mid\left(\omega, a^{\prime}\right) \in B\right\}, a^{\prime} \in A_{-a}$, belong to $\overline{\mathcal{F}}_{a}$. One easily verifies that $\mathcal{W}$ is an extension of the product $\sigma$-algebra $\overline{\mathcal{F}}_{a} \otimes \mathcal{A}_{-a}$ and that $\mathcal{W}$ inherits the Fubini property from $\mathcal{F}_{a}\left(\hat{t}_{a}\right) \otimes \mathcal{A}_{-a}$, i.e. that $\mathcal{W}$ is a Fubini extension of $\overline{\mathcal{F}}_{a} \otimes \mathcal{A}_{-a}$. Finally, the measurability of $\tau_{a}(\cdot, \cdot)$ with respect to $\mathcal{F}_{a}\left(\hat{t}_{a}\right) \otimes \mathcal{A}_{-a}$ and the definition of $\mathcal{F}_{a}$ as the coarsest $\sigma$-algebra with respect to which the function $\omega \rightarrow \boldsymbol{\tau}^{a}(\omega)=\left\{\tau_{a}\left(\omega, a^{\prime}\right)\right\}_{a^{\prime} \in A_{-a}}$ is measurable imply that $\tau_{a}(\cdot, \cdot)$ is also measurable with respect to $\mathcal{W}$.

The Fubini extension of the product $\sigma$-algebra $\overline{\mathcal{F}}_{a} \otimes \mathcal{A}_{-a}$ in Proposition 4.2 need not be rich. Since $\overline{\mathcal{F}}_{a}$ is a coarsening of $\mathcal{F}_{a}\left(t_{a}\right)$, the $\sigma$-algebra $\overline{\mathcal{F}}_{a} \otimes \mathcal{A}_{-a}$ is a coarsening of $\mathcal{F}_{a}\left(\hat{t}_{a}\right) \otimes \mathcal{A}_{-a}$ and need not inherit the richness property of the latter. Indeed, $\overline{\mathcal{F}}_{a} \otimes \mathcal{A}_{-a}$ will not be rich if, e.g., $b_{a}\left(\hat{t}_{a}\right)$ is a degenerata measure that assigns all probability to a singleton $\left\{t_{a^{\prime}}\right\}_{a^{\prime} \in A_{-a}}$. In this case, $\overline{\mathcal{F}}_{a}$ is merely the trivial algebra $\left\{\emptyset, \Omega_{a}\right\}$. However, $\overline{\mathcal{F}}_{a} \otimes \mathcal{A}_{-a}$ will be rich if the specification of $b_{a}\left(\hat{t}_{a}\right)$ involves nontrivial agent-specific uncertainty, so that the measurability of the function $\tau_{a}\left(\cdot, \cdot \mid \hat{t}_{a}\right)$ depends in an essential way on the richness of $\mathcal{F}_{a}\left(\hat{t}_{a}\right) \otimes \mathcal{A}_{-a}$.

\subsection{The Existence of Agent-Specific Priors}

An interpretation of the belief function as a regular conditional distribution presumes that the belief function is measurable. Since I have not introduced a $\sigma$-algebra on the range of the belief function, i.e., the space of probability measures on $\left(R_{a}, \hat{\mathcal{F}}_{a}\right)$, I use the following definition.

Measurability of Belief Functions The belief function $b_{a}(\cdot)$ is said to be measurable if and only if, for any $\hat{\mathcal{F}}_{a}$-measurable function $g$ from $R_{a}$ to $[0,1]$, the function $t_{a} \rightarrow \int_{R_{a}} g(\cdot) d b_{a}\left(\cdot \mid t_{a}\right)$ from $T$ to $[0,1]$ is measurable.

Proposition 4.3 Assume that the belief function $b_{a}(\cdot)$ is coherent and measurable. Then there exists a complete probability space $\left(\Phi, \bar{\Phi}, \bar{\Pi}_{a}\right)$ and there exists a function $\tau$ from $\Phi \times A$ to $T$ such that $\tau$ is measurable with respect to a Fubini extension of the product $\sigma$-algebra $\bar{\Phi} \otimes \mathcal{A}$, and $b_{a}(\tau(\cdot, a))$ is a regular conditional distribution for $\left\{\tau\left(\cdot, a^{\prime}\right)\right\}_{a^{\prime} \in A_{-a}}$ given $\tau(\cdot, a)$.

Proof. Let $\Phi=\Omega_{a} \times T$ and $\Phi=\overline{\mathcal{F}}_{a} \otimes \mathcal{B}(T)$ where $\Omega_{a}$ and $\overline{\mathcal{F}}_{a}$ are given by Proposition 4.2 and $\mathcal{B}(T)$ is the Borel $\sigma$-algebra on $T$. Let $\pi_{a}$ be any 
probability measure on $(T, \mathcal{B}(T))$ and define a measure $\Pi_{a}\left(\pi_{a}\right)$ as the unique measure on $(\Phi, \Phi)$ that satisfies

$$
\Pi_{a}\left(F \times B \mid \pi_{a}\right)=\int_{F} b_{a}\left(F \mid t_{a}\right) d \pi_{a}\left(t_{a}\right)
$$

for $F \in \overline{\mathcal{F}}_{a}$ and $B \in \mathcal{B}(T)$. Further, let $\bar{\Phi}\left(\pi_{a}\right)$ be the $\sigma$-algebra on $\Phi$ that is induced by $\Phi$ and the class of subsets of sets in $\Phi$ that have $\Pi_{a}\left(\pi_{a}\right)$ measure zero, and let $\bar{\Pi}_{a}\left(\pi_{a}\right)$ be the extension of $\Pi_{a}\left(\pi_{a}\right)$ to $\bar{\Phi}\left(\pi_{a}\right)$, i.e. the completion of $\Pi_{a}\left(\pi_{a}\right)$. Then by construction, $\left(\Phi, \bar{\Phi}\left(\pi_{a}\right), \bar{\Pi}_{a}\left(\pi_{a}\right)\right)$ is a complete probability space.

Next, let $\tau: \Phi \times A \rightarrow T$ be such that, for any $\left(\omega, t_{a}, a^{\prime}\right) \in \Phi \times A=$ $\Omega_{a} \times T \times A$,

$$
\tau(\omega, t, \bar{a})=t_{a} \quad \text { if } \quad a^{\prime}=a
$$

and

$$
\tau\left(\omega, t_{a}, a^{\prime}\right)=\tau_{a}\left(\omega, a^{\prime}\right) \text { if } \quad a^{\prime} \neq a
$$

where $\tau_{a}(\cdot, \cdot)$ is given by Proposition 4.2.

To obtain the claimed measurability property of $\tau$, consider the class $\mathcal{V}$ of sets $V \subset \Phi \times A=\Omega_{a} \times T \times A$ that satisfy the following conditions:

$$
\begin{gathered}
\operatorname{Proj}_{T} V \in \mathcal{B}(T), \\
\operatorname{Proj}_{\Omega_{a} \times A} V \cap\left[\Omega_{a} \times\{a\}\right] \in \overline{\mathcal{F}}_{a} \otimes\{a\},
\end{gathered}
$$

and

$$
\operatorname{Proj}_{\Omega_{a} \times A} V \cap\left[\Omega_{a} \times A_{-a}\right] \in \mathcal{W}
$$

where $\mathcal{W}$ is the Fubini extension of the product $\sigma$-algebra $\overline{\mathcal{F}}_{a} \otimes \mathcal{A}_{-a}$ with respect to which the function $\tau_{a}(\cdot, \cdot)$ in Proposition 4.2 is measurable. One easily verifies that $\mathcal{V}$ is a Fubini extension of the product $\sigma$-algebra $\bar{\Phi} \otimes \mathcal{A}_{-a}$. Measurability of the function $\tau$ with respect to $\mathcal{V}$ follows from (4.4) - (4.8) and the measurability of the function $\tau_{a}(\cdot, \cdot)$ with respect to $\mathcal{W}$.

Finally, (4.3), (4.4), and (4.5) imply that, for $\pi_{a}$-almost all $t_{a} \in T, b_{a}\left(t_{a}\right)$ is a conditional distribution for $\left\{\tau\left(\cdot, a^{\prime}\right)\right\}_{a^{\prime} \in A_{-a}}$ given $\tau(\cdot, a)$.

In the preceding proof, the choice of the distribution $\pi_{a}$ on $(T, \mathcal{B}(T))$ is arbitrary. This observation yields:

Remark 4.4 The measure $\bar{\Pi}_{a}$ in Proposition 4.3 can be chosen to have any marginal distribution on the factor $T$ in the product $\Phi=\Omega_{a} \times T$. 
Propositions 4.2 and 4.3 show that, if the belief function $b_{a}(\cdot)$ of agent $a$ is coherent as well as measurable, it can be interpreted as a result of the agent's conditioning on the observation of his own type in a model with an agent-specific prior. The requirement of coherence is restrictive, but I do not see any way to eliminate it without losing the property that the probability spaces $\left(\Omega_{a}\left(t_{a}\right), \mathcal{F}_{a}\left(t_{a}\right), P_{a}\left(t_{a}\right)\right)$ that underlie the specification of the beliefs $b_{a}\left(t_{a}\right)$ for $t_{a} \in T$ are all complete, or equivalently the property that the probability spaces $\left(R_{\boldsymbol{\tau}^{a}\left(t_{a}\right)}, \hat{\mathcal{F}}_{a}\left(t_{a}\right), b_{a}\left(t_{a}\right)\right), t_{a} \in T$, are all complete. As indicated by Lemma 4.1, the property of coherence ensures that the measures $b_{a}\left(t_{a}\right), t_{a} \in T$, all have the same domains and the same null sets. Without this property, say if there are two types $t_{a}$ and $\hat{t}_{a}$ with $\hat{\mathcal{F}}_{a}\left(t_{a}\right) \neq \hat{\mathcal{F}}_{a}\left(\hat{t}_{a}\right)$, extending the measure $b_{a}\left(t_{a}\right)$ to the union $\hat{\mathcal{F}}_{a}\left(t_{a}\right) \cup \hat{\mathcal{F}}_{a}\left(\hat{t}_{a}\right)$ and then taking the completion would seem to run afoul of the fact any sufficiently rich space will have nonmeasurable sets.

\subsection{Agent-Specific Priors and Anonymity in Beliefs}

In Section 3.2 above, I introduced anonymity in beliefs as a property of the measure $b_{a}\left(t_{a}\right)$ where $t_{a}$ was taken as given. I now consider the implications of this property for an agent-specific prior that induces the belief function $b_{a}(\cdot)$ as a regular conditional distribution.

Proposition 4.5 Assume that the belief function $b_{a}(\cdot)$ is coherent and measurable. Let $\left(\Phi, \bar{\Phi}, \bar{\Pi}_{a}\right)$ and $\tau: \Phi \times A \rightarrow T$ be such that the probability space $\left(\Phi, \bar{\Phi}, \bar{\Pi}_{a}\right)$ is complete, the mapping $\tau$ is measurable with respect to a Fubini extension of the $\sigma$-algebra $\bar{\Phi} \otimes \mathcal{A}$, and $b_{a}(\tau(\cdot, a))$ is a regular conditional distribution for $\left\{\tau\left(\cdot, a^{\prime}\right)\right\}_{a^{\prime} \in A_{-a}}$ given $\tau(\cdot, a)$. Then the following statements are equivalent:

(a) For all $\varphi \in \Phi, b_{a}(\tau(\varphi, a))$ satisfies anonymity in beliefs.

(b) Under the measure $\bar{\Pi}_{a}$, the random variables $\tau\left(\cdot, a^{\prime}\right), a^{\prime} \in A_{-a}$, are essentially pairwise exchangeable, i.e., there exists a probability measure $p_{a}(\cdot)$ on $T^{2}$ such that, for $\alpha_{-a^{-}}$almost all $a^{1} \in A_{-a}$,

$$
\bar{\Pi}_{a}\left(\tau\left(\cdot, a^{1}\right)^{-1}\left(B_{1}\right) \cap \tau\left(\cdot, a^{2}\right)^{-1}\left(B_{2}\right)\right)=p_{a}\left(B_{1} \times B_{2}\right)=p_{a}\left(B_{2} \times B_{1}\right)
$$

for $\alpha_{-a}$-almost all $a^{2} \in A_{-a}$ and all Borel sets $B_{1}, B_{2} \subset T$.

(c) If $\mathcal{D} \subset \bar{\Phi}$ is the Borel $\sigma$-algebra that is generated be the mapping $\varphi \rightarrow$ $D\left(\left\{\tau\left(\varphi, a^{\prime}\right)\right\}_{a^{\prime} \in A_{-a}}\right)$, then conditionally on $\mathcal{D}$, the random variables $\tau\left(\cdot, a^{\prime}\right)$, $a^{\prime} \in A_{-a}$, are essentially pairwise conditionally independent and identically distributed. Moreover, the sample distribution $D\left(\left\{\tau\left(\varphi, a^{\prime}\right)\right\}_{a^{\prime} \in A_{-a}}\right)$ of 
types in the population is almost surely equal to the common conditonal distribution of types in the population, i..e., for $\alpha_{-a}$ almost all $a^{1} \in A_{-a}$, $D\left(\left\{\tau\left(\cdot, a^{\prime}\right)\right\}_{a^{\prime} \in A_{-a}}\right)$ is a regular conditional distribution for $\tau\left(\cdot, a^{1}\right)$ given $\mathcal{D}$.

Proof. I first prove that (a) implies (b). The left-hand side of (4.9) can be written in the form

$$
\begin{aligned}
\bar{\Pi}_{a}\left(\left\{\varphi \mid \tau\left(\varphi, a^{1}\right)\right.\right. & \left.\left.\in B_{1}\right\} \cap\left\{\varphi \mid \tau\left(\varphi, a^{2}\right) \in B_{2}\right\}\right) \\
& =\int_{\Phi} \chi_{B_{1}}\left(\tau\left(\varphi, a^{1}\right)\right) \cdot \chi_{B_{2}}\left(\tau\left(\varphi, a^{2}\right)\right) d \bar{\Pi}_{a}(\varphi)
\end{aligned}
$$

where $\chi_{B_{1}}: T \rightarrow\{0,1\}$ and $\chi_{B_{2}}: T \rightarrow\{0,1\}$ are the characteristic functions of the sets $B_{1}$ and $B_{2}$, i.e., for $i=1,2, \chi_{B_{i}}\left(t^{i}\right)=1$ if $\left(t, t^{i}\right) \in B_{i}$ and $\chi_{B_{i}}\left(t^{i}\right)=0$ if $t^{i} \notin B_{i}$. It therefore suffices to show that, if the measure $b_{a}(\tau(\varphi, a))$ satisfies anonymity in beliefs for $\bar{\Pi}_{a}$-almost all $\varphi \in \Phi$, then

$$
\int_{\Phi} g\left(\tau\left(\varphi, a^{1}\right), \tau\left(\varphi, a^{2}\right)\right) d \bar{\Pi}_{a}(\varphi)=\int_{\Phi} g\left(\tau\left(\varphi, a^{2}\right), \tau\left(\varphi, a^{1}\right)\right) d \bar{\Pi}_{a}(\varphi)
$$

for all measurable functions $g: T^{2} \rightarrow[0,1]$.

For any measurable functions $g: T^{2} \rightarrow[0,1]$. the fact that $b_{a}(\tau(\cdot, a))$ is a regular conditional distribution for $\left\{\tau\left(\cdot, a^{\prime}\right)\right\}_{a^{\prime} \in A_{-a}}$ given $\tau(\cdot, a)$ implies that the left-hand side of (4.11) is equal to

$$
\int_{\Phi} \int_{R_{a}} g\left(t_{a^{1}}, t_{a^{2}}\right) d b\left(\left\{t_{a^{\prime}}\right\}_{a^{\prime} \in A_{-a}} \mid \tau(\varphi, a)\right) d \bar{\Pi}_{a}(\varphi) .
$$

By Proposition 3.3, statement (a) implies that (4.12) can be rewritten in the form

$$
\int_{\Phi} \int_{\mathcal{M}(T)} \int_{T} \int_{T} g\left(t_{a^{1}}, t_{a^{2}}\right) d \delta\left(t_{a^{1}}\right) d \delta\left(t_{a^{2}}\right) d b^{*}(\delta \mid \tau(\varphi, a)) d \bar{\Pi}_{a}(\varphi) .
$$

By Fubini's theorem, (4.13) in turn is equal to

$$
\int_{\Phi} \int_{\mathcal{M}(T)} \int_{T} \int_{T} g\left(t_{a^{1}}, t_{a^{2}}\right) d \delta\left(t_{a^{2}}\right) d \delta\left(t_{a^{1}}\right) d b^{*}(\delta \mid \tau(\varphi, a)) d \bar{\Pi}_{a}(\varphi) .
$$

By a relabeling of indices, finally, (4.14) can be rewritten as

$$
\int_{\Phi} \int_{\mathcal{M}(T)} \int_{T} \int_{T} g\left(t_{a^{2}}, t_{a^{1}}\right) d \delta\left(t_{a^{1}}\right) d \delta\left(t_{a^{2}}\right) d b^{*}(\delta \mid \tau(\varphi, a)) d \bar{\Pi}_{a}(\varphi)
$$

which is easily seen to be equal to the right-hand side of (4.11). 
Next, if (b) holds, then (c) follows from Proposition 3 of Qiao et al. (2016).

Finally, assume that (c) holds. Let $\mathcal{C}$ be the $\sigma$-algebra that is generated by the mapping

$$
\varphi \rightarrow\left(\tau(\varphi, a), D\left(\left\{\tau\left(\varphi, a^{\prime}\right)\right\}_{a^{\prime} \in A_{-a}}\right)\right)
$$

For any $a^{1} \in A_{-a}$ and $a^{2} \in A_{-a}$, let $\mu^{a^{1} a^{2}}(\cdot)$ be a regular conditional distribution for the pair $\left(\tau\left(\cdot, a^{1}\right), \tau\left(\cdot, a^{2}\right)\right)$ given $\mathcal{C}$ and let

$$
\left.\Phi^{a^{1} a^{2}}:=\left\{\varphi \in \Phi \mid \mu^{a^{1} a^{2}}(\varphi)\right)=\left(D\left(\left\{\tau\left(\varphi, a^{\prime}\right)\right\}_{a^{\prime} \in A_{-a}}\right)\right)^{2}\right\}
$$

is the set of states $\varphi$ for which $\mu^{a^{1} a^{2}}(\varphi)$ is equal to the product distribution $\left(D\left(\left\{\tau\left(\varphi, a^{\prime}\right)\right\}_{a^{\prime} \in A_{-a}}\right)\right)^{2}$. I claim that, for $\alpha_{-a^{-}}$-almost all $a^{1} \in A_{-a}$, one may therefore infer that $\bar{\Pi}_{a}\left(\Phi^{a^{1} a^{2}}\right)=1$ for $\alpha_{-a}$-almost all $a^{2} \in A_{-a}$.

Because $T$ is a complete separable metric space, $\mathcal{M}(T)$ is also a complete separable metric space. By standard arguments, therefore $\mathcal{C}$ is countably generated. Trivially also, $\mathcal{D} \subset \mathcal{C} \subset \bar{\Phi}$. By Proposition 3 of Hammond and Sun (2006) therefore, (c) implies that, conditionally on $\mathcal{C}$, the random variables $\tau\left(\cdot, a^{\prime}\right), a^{\prime} \in A_{-a}$, are essentially pairwise conditionally independent. By the argument given in the proof of Proposition 3 in Hammond and Sun (2006), (c) also implies that, conditionally on $\mathcal{C}$, the random variables $\tau\left(\cdot, a^{\prime}\right)$, $a^{\prime} \in A_{-a}$, are essentially identically distributed and that, for $\alpha_{-a^{-}}$-almost all $a^{\prime \prime} \in A_{-a}, D\left(\left\{\tau\left(\cdot, a^{\prime}\right)\right\}_{a^{\prime} \in A_{-a}}\right)$ is a regular conditional distribution for $\tau\left(\cdot, a^{\prime \prime}\right)$ given $\mathcal{C}$. The claim that, for $\alpha_{-a^{-a l m o s t}}$ all $a^{1} \in A_{-a}, \bar{\Pi}_{a}\left(\Phi^{a^{1} a^{2}}\right)=1$ for $\alpha_{-a^{-}}$-almost all $a^{2} \in A_{-a}$ follows immediately.

Because $b_{a}(\tau(\cdot, a))$ is a regular conditional distribution for $\left\{\tau\left(\cdot, a^{\prime}\right)\right\}_{a^{\prime} \in A_{-a}}$ given $\tau(\cdot, a), \bar{\Pi}_{a}\left(\Phi^{a^{1} a^{2}}\right)=1$ implies

$$
\int b_{a}\left\{\left\{t_{a^{\prime}}\right\}_{a^{\prime} \in A_{-a}} \mid \mu^{a^{1} a^{2}}(\varphi)=D\left(\left\{t_{a^{\prime}}\right\}_{a^{\prime} \in A_{-a}}\right)^{2}\right\} \mid \tau(\varphi, a) d \bar{\Pi}_{a}(\varphi)=1 .
$$

Because $\mu^{a^{1} a^{2}}(\cdot)$ is measurable with respect to $\mathcal{C}$, there exists a function $\hat{\mu}^{a^{1} a^{2}}(\cdot)$ such that

$$
\mu^{a^{1} a^{2}}(\varphi)=\hat{\mu}^{a^{1} a^{2}}\left(\tau(\varphi, a), D\left(\left\{\tau\left(\varphi, a^{\prime}\right)\right\}_{a^{\prime} \in A_{-a}}\right)\right)
$$

for $\bar{\Pi}_{a}$-almost all $\varphi$. Moreover, for $\bar{\Pi}_{a}$-almost all $\varphi$, (c) implies

$$
D\left(\left\{t_{a^{\prime}}\right\}_{a^{\prime} \in A_{-a}}\right)=D\left(\left\{\tau\left(\varphi, a^{\prime}\right)\right\}_{a^{\prime} \in A_{-a}}\right)
$$


for $b_{a}(\tau(\varphi, a))$-almost all $\left\{t_{a^{\prime}}\right\}_{a^{\prime} \in A_{-a}}$. Thus (4.17) implies

$\int b_{a}\left\{\left\{t_{a^{\prime}}\right\}_{a^{\prime} \in A_{-a}} \mid \hat{\mu}^{a^{1} a^{2}}\left(\tau(\varphi, a), D\left(\left\{t_{a^{\prime}}\right\}_{a^{\prime} \in A_{-a}}\right)\right)=D\left(\left\{t_{a^{\prime}}\right\}_{a^{\prime} \in A_{-a}}\right)^{2}\right\} \mid \tau(\varphi, a) d \bar{\Pi}_{a}(\varphi)=1$

and therefore

$b_{a}\left(\left\{\left\{t_{a^{\prime}}\right\}_{a^{\prime} \in A_{-a}}\left|\hat{\mu}^{a^{1} a^{2}}\left(\tau(\varphi, a), D\left(\left\{t_{a^{\prime}}\right\}_{a^{\prime} \in A_{-a}}\right)\right)=\left(D\left(\left\{t_{a^{\prime}}\right\}_{a^{\prime} \in A_{-a}}\right)^{2}\right\}\right| \tau(\varphi, a)\right)=1\right.$

for $\bar{\Pi}_{a}$-almost all $\varphi$. Because the belief function $b_{a}(\cdot)$ is coherent, it follows that

$$
b_{a}\left(\left\{\left\{t_{a^{\prime}}\right\}_{a^{\prime} \in A_{-a}}\left|\hat{\mu}^{a^{1} a^{2}}\left(t_{a}, D\left(\left\{t_{a^{\prime}}\right\}_{a^{\prime} \in A_{-a}}\right)\right)=\left(D\left(\left\{t_{a^{\prime}}\right\}_{a^{\prime} \in A_{-a}}\right)^{2}\right\}\right| t_{a}\right)=1\right.
$$

for all $t_{a} \in T$.

Thus, if (c) holds, then for $\alpha_{-a^{-}}$-almost all $a^{1} \in A_{-a}$, (4.20) holds for $\alpha_{-a}$-almost all $a^{2} \in A_{-a}$, for all $t_{a} \in T$. By Proposition 3.3, it follows that, for all $t_{a} \in T, b_{a}\left(t_{a}\right)$ satisfies anonymity in beliefs.

Thus at the level of priors, anonymity in beliefs translates into a condition of essential pairwise exchangeability and the accompanying conditional law of large numbers.

Proposition 4.5 assumes, and the proof uses, the property of coherence of the belief function $b_{a}(\cdot)$. However, this property is less important here than in Proposition 4.3 above. In the proof that (c) implies (a), coherence yields the conclusion that $b_{a}\left(t_{a}\right)$ satisfies anonymity in beliefs for all $t_{a}$. Without coherence, an admissible commutation of "almost all" quantifiers would yield the conclusion that $b_{a}(\tau(\varphi, a))$ satisfies anonymity in beliefs for $\bar{\Pi}_{a}$-almost all $\varphi$. As is evident from the proof, this weakening of statement (a) does not affect the validity of the implication that (a) implies (b).

Which version of the result one prefers, depends on which interpretation of the belief function $b_{a}(\cdot)$ one considers appropriate. If one thinks of the beliefs $b_{a}\left(t_{a}\right), t_{a} \in T$, as the "real" representation of the agent's expectations, and of the prior $\bar{\Pi}_{a}$ as a derived concept, one is likely to prefer the version of the result given here, which involves anonymity in beliefs for all $t_{a}$. In contrast, if one thinks of the prior $\bar{\Pi}_{a}$ as the "real" representation of the agent's expectations, and of the belief function $b_{a}(\cdot)$ as a derived concept, one is likely to prefer the version of the result that involves anonymity in beliefs for $\bar{\Pi}_{a}$-almost all $\tau(\varphi, a)$ and that does not require coherence. 


\subsection{Macro Belief Functions and Agent-Specific Priors}

A belief function $b_{a}(\cdot)$ that satisfies anonymity in beliefs is fully characterized by the associated macro belief function $b_{a}^{*}(\cdot)$ where $b_{a}^{*}\left(t_{a}\right)=b_{a}\left(t_{a}\right) \circ D(\cdot)^{-1}$ for all $t_{a}$. In Proposition 3.6 above, I had shown that, for any type $t_{a}$ of agent $a$, any measure $\beta$ on $\mathcal{M}(T)$, and any rich Fubini extension $\left(\Omega_{a}\left(t_{a}\right) \times\right.$ $\left.A_{-a}, \mathcal{F}_{a}\left(t_{a}\right) \otimes \mathcal{A}_{-a}, P_{a}\left(t_{a}\right) \otimes \alpha_{-a}\right)$, there exists an $\mathcal{F}_{a}\left(t_{a}\right) \otimes \mathcal{A}_{-a}$-measurable mapping $\tau$ from $\Omega_{a}\left(t_{a}\right) \times A_{-a}$ to $T$ such that the associated belief $b_{a}\left(t_{a}\right)$ satisfies anonymity in beliefs and the macro belief $b_{a}^{*}\left(t_{a}\right)$ is equal to $\beta$, i.e. once richness of the Fubini extension is imposed, there is no restriction on the scope of macro uncertainty. In the following, I establish an analogous result for macro belief functions and agent-specific priors. The construction is more complicated than in Proposition 3.6 because, as in Proposition 4.3, there is a need to ensure that the beliefs of different types are coherent and measurable.

Coherence of Macro Belief Functions The macro belief function $b_{a}^{*}(\cdot)$ is said to be coherent if, for any $t_{a}$ and $\hat{t}_{a}$ in $T$, the probability measures $b_{a}^{*}\left(t_{a}\right)$ and $b_{a}^{*}\left(\hat{t}_{a}\right)$ are mutually absolutely continuous, i.e., any Borel set $B \subset \mathcal{M}(T)$ satisfies $b_{a}^{*}\left(B \mid t_{a}\right)>0$ if and only if it also satisfies $b_{a}^{*}\left(B \mid \hat{t}_{a}\right)>0$.

Measurability of Macro Belief Functions The macro belief function $b_{a}^{*}(\cdot)$ is said to be measurable if, for any Borel set $B \subset \mathcal{M}(T)$, the set of types with macro beliefs in $B$, i.e. the set $\left\{t_{a} \in T \mid b_{a}^{*}\left(t_{a}\right) \in B\right\}$, is itself a Borel subset of $T$.

Given that, for any $B \subset \mathcal{M}(T)$, the equation $b_{a}^{*}\left(t_{a}\right)=b_{a}\left(t_{a}\right) \circ D(\cdot)^{-1}$ implies

$$
b_{a}^{*}\left(B \mid t_{a}\right)=b_{a}\left(\left\{\left\{t_{a^{\prime}}\right\}_{a^{\prime} \in A_{-a}} \mid D\left(\left\{t_{a^{\prime}}\right\}_{a^{\prime} \in A_{-a}}\right) \in B\right\} \mid t_{a}\right),
$$

one easily verifies that, if the belief function $b_{a}(\cdot)$ is coherent, the associated macro belief function $b_{a}^{*}(\cdot)$ is also coherent. Similarly, it is easy to verify that, if $b_{a}(\cdot)$ is measurable, the associated macro belief function $b_{a}^{*}(\cdot)$ is also measurable. As a partial converse to these observations, the following result shows, that, starting from a coherent and measurable function $\beta(\cdot)$ from $T$ to $\mathcal{M}(\mathcal{M}(T)$ ), one can always find a coherent and measurable belief function $b_{a}(\cdot)$ that has $\beta(\cdot)$ as its macro belief function.

Proposition 4.6 Let $\beta(\cdot)$ be any coherent and measurable function from $T$ to $\mathcal{M}(\mathcal{M}(T))$. Let $\left(\Phi, \bar{\Phi}, \bar{\Pi}_{a}\right)$ be a complete probability space. If the Fubini extension $\left(\Phi \times A, \bar{\Phi} \otimes \mathcal{A}, \bar{\Pi}_{a} \otimes \alpha\right)$ is rich, there exists a mapping $\tau: \Phi \times A \rightarrow T$ 
that is measurable with respect to $\bar{\Phi} \otimes \mathcal{A}$ and a coherent and measurable belief function $b_{a}(\cdot)$ such that $b_{a}(\tau(\cdot, a))$ is a regular conditional distribution for $\left\{\tau\left(\cdot, a^{\prime}\right)\right\}_{a^{\prime} \in A_{-a}}$ given $\tau(\cdot, a)$, and, moreover, for all $t_{a} \in T, b_{a}\left(t_{a}\right)$ satisfies anonymity in beliefs and the associated macro belief $b_{a}^{*}\left(t_{a}\right)$ coincides with $\beta\left(t_{a}\right)$.

Proof. The argument is similar to the one in the proof of Proposition 3.6, with $\left(\Omega_{a}\left(t_{a}\right), \mathcal{F}_{a}\left(t_{a}\right), P_{a}\left(t_{a}\right)\right)$ replaced by $\left(\Phi, \bar{\Phi}, \bar{\Pi}_{a}\right)$. Given the function $\beta(\cdot)$, let $\Psi_{\beta} \in \mathcal{M}(T \times \mathcal{M}(T))$ be such that, for some (arbitrary) marginal distribution $\Psi_{\beta}^{T}$ on $T$, one has

$$
\Psi_{\beta}\left(B_{t} \times B_{\delta}\right)=\int_{B_{t}} \beta\left(B_{\delta} \mid t_{a}\right) d \Psi_{\beta}^{T}\left(t_{a}\right)
$$

for all Borel sets $B_{t} \subset T$ and $B_{\delta} \subset \mathcal{M}(T)$. By standard arguments, there exists a pair $\left(\tau_{\beta}(\cdot, a), \tilde{\delta}_{\beta}(\cdot)\right)$ of random variables on $\left(\Phi, \bar{\Phi}, \bar{\Pi}_{a}\right)$, taking values in $T \times \mathcal{M}(T)$, such that

$$
\bar{\Pi}_{a} \circ\left(\tau_{\beta}(\cdot, a), \tilde{\delta}_{\beta}(\cdot)\right)^{-1}=\Psi_{\beta},
$$

i.e. the probability distribution of $\left(\tau_{\beta}(\cdot, a), \tilde{\delta}_{\beta}(\cdot)\right)$ is $\Psi_{\beta}$.

As in the proof of Proposition 3.6, let $f$ be a measurable function from $\mathcal{M}(T) \times[0,1]$ into $T$ such that for any $\delta \in \mathcal{M}(T)$,

$$
\ell \circ f(\delta, \cdot)^{-1}=\delta
$$

where $\ell$ is the uniform distribution on $[0,1]$. Given this function $f$ and the random pair $\left(\tau_{\beta}(\cdot, a), \tilde{\delta}_{\beta}(\cdot)\right)$, define a mapping $\tau(\cdot, \cdot)$ from $\Omega_{a}\left(t_{a}\right) \times A$ into $T$ by setting

$$
\begin{aligned}
\tau(\cdot, a)= & \tau_{\beta}(\cdot, a) \\
& \text { and } \\
\tau\left(\cdot, a^{\prime}\right)= & f\left(\tilde{\delta}_{\beta}(\cdot), h\left(\cdot, a^{\prime}\right)\right) \text { for } a^{\prime} \in A_{-a},
\end{aligned}
$$

where $h$ is the function given by the richness of the Fubini extension $(\Phi \times$ $\left.A, \bar{\Phi} \otimes \mathcal{A}, \bar{\Pi}_{a} \otimes \alpha\right)$. Given that $\tau(\cdot, \cdot)$ is measurable with respect to $\bar{\Phi} \otimes \mathcal{A}_{-a}$, one easily verifies that $\tau(\cdot, \cdot)$ is measurable with respect to $\bar{\Phi} \otimes \mathcal{A}$, as claimed in the proposition.

For any $t_{a} \in T$, set $\left.b_{a}\left(t_{a}\right)=\int_{\mathcal{M}(T)} \hat{b}_{a}(\delta) d \beta\left(\delta \mid t_{a}\right)\right)$ where $\hat{b}_{a}$ is the function given by Remark 3.7. In the remainder of the proof, I will show that the function $b_{a}$ satisfies the claims made in the proposition. I first show 
that $b_{a}(\tau(\cdot, a))$ is a regular conditional distribution for $\left\{\tau\left(\cdot, a^{\prime}\right)\right\}_{a^{\prime} \in A_{-a}}$ given $\tau(\cdot, a)$.

Let $\mathcal{C}_{\delta}$ be the sub- $\sigma$-algebra of $\bar{\Phi}$ that is generated by the random variable $\tilde{\delta}_{\beta}(\cdot)$. Because $\mathcal{M}(T)$ is a complete separable metric space, $\mathcal{C}_{\tilde{\delta}}$ is countably generated. Because the random variables $h\left(\cdot, a^{\prime}\right), a^{\prime} \in A_{-a}$, are essentially pairwise independent, Proposition 3 in Hammond and Sun (2006) implies that they are also essentially pairwise conditionally independent given $\mathcal{C}_{\tilde{\delta}}$. As in Remark 1 of Hammond and Sun (2008), it follows that the random pairs $\left(\tilde{\delta}(\cdot), h\left(\cdot, a^{\prime}\right)\right), a^{\prime} \in A_{-a}$, are also essentially pairwise conditionally independent given $\mathcal{C}_{\tilde{\delta}}$ and so are the random variables $\tau\left(\cdot, a^{\prime}\right)=f\left(\tilde{\delta}(\cdot), h\left(\cdot, a^{\prime}\right)\right)$, $a^{\prime} \in A_{-a}$. By Proposition 3 in Qiao et al. (2016), these random variables are also essentially pairwise exchangeable.

Because, for $\alpha_{-a^{-}}$-almost every $a^{\prime} \in A_{-a}$, the random variable $h\left(\cdot, a^{\prime}\right)$ has the uniform distribution $\ell,(4.23)$ and (4.25) imply that, for $\alpha_{-a}$-almost every $a^{\prime} \in A_{-a}$, conditional on the event $\tilde{\delta}_{\beta}(\cdot)=\delta$, the probability distribution of $\tau_{a}\left(\cdot, a^{\prime}\right)$ is almost surely equal to $\delta$. For $\alpha_{-a}$-almost every $a^{\prime} \in A_{-a}$, therefore the function $\tilde{\delta}_{\beta}(\cdot)$ is a regular conditional distribution for the random variable $\tau_{a}\left(\cdot, a^{\prime}\right)$ given $\mathcal{C}_{\delta}$.

Next, let $\mathcal{C}_{\tau \delta}$ be the sub- $\sigma$-algebra of $\bar{\Phi}$ that is generated by the pair $\left(\tau_{\beta}(\cdot, a), \tilde{\delta}_{\beta}(\cdot)\right)$. Then $\mathcal{C}_{\tau \tilde{\delta}}$ is also countably generated, and $\mathcal{C}_{\delta} \subset \mathcal{C}_{\tau \delta}$. By another application of Proposition 3 in Hammond and Sun (2006), it follows that the random variables $\tau\left(\cdot, a^{\prime}\right)=f\left(\tilde{\delta}(\cdot), h\left(\cdot, a^{\prime}\right)\right), a^{\prime} \in A_{-a}$, are also essentially pairwise conditionally independent given $\mathcal{C}_{\tau \delta}$. Moreover, by the argument in the proof of Proposition 3 of Hammond and Sun (2006), for almost every $a^{\prime} \in A_{-a}$, the function $\tilde{\delta}_{\beta}(\cdot)$ is also a regular conditional distribution for the random variable $\tau_{a}\left(\cdot, a^{\prime}\right)$ given $\mathcal{C}_{\tau \delta} . \tilde{\delta}$ is a regular conditional distribution for $\tau\left(\cdot, a^{\prime}\right)$ given $\mathcal{C}_{\tau \delta}$. 
For any measurable function $g$ on $T \times R_{a}$, we therefore obtain

$$
\begin{aligned}
& \int_{\Phi} g\left(\tau(\varphi, a),\left\{\tau\left(\varphi, a^{\prime}\right)\right\}_{a^{\prime} \in A_{-a}}\right) d \bar{\Pi}_{a}(\varphi) \\
= & \int_{\Phi} \int_{R_{a}} g\left(\tau(\varphi, a),\left\{t_{a^{\prime}}\right\}_{a^{\prime} \in A_{-a}}\right) d \hat{b}_{a}\left(\left\{t_{a^{\prime}}\right\}_{a^{\prime} \in A_{-a}} \mid \tilde{\delta}_{\beta}(\varphi)\right) d \bar{\Pi}_{a}(\varphi) \\
= & \int_{T \times \mathcal{M}(T)} \int_{R_{a}} g\left(t_{a},\left\{t_{a^{\prime}}\right\}_{a^{\prime} \in A_{-a}}\right) d \hat{b}_{a}\left(\left\{t_{a^{\prime}}\right\}_{a^{\prime} \in A_{-a}} \mid \delta\right) d \Psi_{\beta}\left(t_{a}, \delta\right) \\
= & \int_{T} \int_{\mathcal{M}(T)} \int_{R_{a}} g\left(t_{a},\left\{t_{a^{\prime}}\right\}_{a^{\prime} \in A_{-a}}\right) d \hat{b}_{a}\left(\left\{t_{a^{\prime}}\right\}_{a^{\prime} \in A_{-a}} \mid \delta\right) d \beta\left(\delta \mid t_{a}\right) d \Psi_{T}\left(t_{a}\right) \\
= & \int_{\Phi} \int_{\mathcal{M}(T)} \int_{R_{a}} g\left(\tau(\varphi, a),\left\{t_{a^{\prime}}\right\}_{a^{\prime} \in A_{-a}}\right) d \hat{b}_{a}\left(\left\{t_{a^{\prime}}\right\}_{a^{\prime} \in A_{-a}} \mid \delta\right) d \beta(\delta \mid \tau(\varphi, a)) d \bar{\Pi}_{a}(\varphi) \\
= & \int_{\Phi} \int_{\mathcal{M}(T)} \int_{R_{a}} g\left(\tau(\varphi, a),\left\{t_{a^{\prime}}\right\}_{a^{\prime} \in A_{-a}}\right) d b_{a}\left(\left\{t_{a^{\prime}}\right\}_{a^{\prime} \in A_{-a}} \mid \tau(\varphi, a)\right) d \bar{\Pi}_{a}(\varphi),
\end{aligned}
$$

which proves that $b_{a}(\tau(\cdot, a))$ is in fact a regular conditional distribution for $\left\{\tau\left(\cdot, a^{\prime}\right)\right\}_{a^{\prime} \in A_{-a}}$ given $\tau(\cdot, a)$.

Turning to the other claims in the proposition, coherence and measurability of $b_{a}$ follow from the coherence and measurability of $\beta$. Because, conditionally on $\mathcal{C}_{\tau \delta}$, the random variables $\tau_{a}\left(\cdot, a^{\prime} \mid t_{a}\right), a^{\prime} \in A_{-a}$, are essentially pairwise independent, Corollary 2 of Qiao et al. (2016) implies that

$$
D\left(\left\{\tau\left(\varphi, a^{\prime}\right)\right\}_{a^{\prime} \in A_{-a}}\right)=\tilde{\delta}_{\beta}(\varphi),
$$

$\bar{\Pi}_{a}$-almost surely, and therefore that the macro belief $b_{a}^{*}\left(t_{a}\right)$ coincides with $\beta\left(t_{a}\right)$ for all $t_{a}$. Finally, anonymity in beliefs follows from the essential pairwise exchangeability of the random variables $\tau\left(\cdot, a^{\prime}\right), a^{\prime} \in A_{-a}$.

\section{Common Priors}

\subsection{Common Priors with Anonymity in Beliefs}

The incomplete-information model $\left\{T, \Theta, \theta_{a}, b_{a}\right\}_{a \in A}$ is said to admit a common prior if there exist a complete probability space $(\Phi, \bar{\Phi}, \bar{\Pi})$ and a mapping $\tau: \Phi \times A \rightarrow T$ that is measurable with respect to a Fubini extension of the $\sigma$-algebra $\bar{\Phi} \otimes \mathcal{A}$ such that for $\alpha$-almost all $a \in A, b_{a}(\tau(\cdot, \varphi))$ is a regular conditional distribution for $\left\{\tau\left(\cdot, a^{\prime}\right)\right\}_{a^{\prime} \in A_{-a}}$ given $\tau(\cdot, \varphi)$.

In the following, I discuss the existence of a common prior in the incompleteinformation model $\left\{T, \Theta, \theta_{a}, b_{a}\right\}_{a \in A}$. As is well known, even in models with 
finitely many agents, the existence of a common prior cannot be taken for granted. ${ }^{15}$ The conditions on the belief system under which a common prior exists in models with finitely many agents are very restrictive, the more so the more agents there are. ${ }^{16}$

Having nothing to say on the general case, I focus on the case where the beliefs $b_{a}(\tau(\varphi, a))$ satisfy the condition of anonymity in beliefs. The following result is an immediate consequence of Proposition 4.5.

Proposition 5.1 Assume that the belief functions $b_{a}(\cdot), a \in A$, are coherent and measurable. Let $(\Phi, \bar{\Phi}, \bar{\Pi})$ and $\tau: \Phi \times A \rightarrow T$ be such that the probability space $(\Phi, \bar{\Phi}, \bar{\Pi})$ is complete, the mapping $\tau$ is measurable with respect to a Fubini extension of the $\sigma$-algebra $\bar{\Phi} \otimes \mathcal{A}$, and $b_{a}(\tau(\cdot, a))$ is a regular conditional distribution for $\left\{\tau\left(\cdot, a^{\prime}\right)\right\}_{a^{\prime} \in A_{-a}}$ given $\tau(\cdot, a)$. Then the following statements are equivalent:

(a) For $\alpha$-almost $a \in A$ and all $\varphi \in \Phi, b_{a}(\tau(\varphi, a))$ satisfies anonymity in beliefs.

(b) Under the measure $\bar{\Pi}$, the random variables $\tau\left(\cdot, a^{\prime}\right), a^{\prime} \in A$, are essentially pairwise exchangeable, i.e., there exists a probability measure $p_{a}(\cdot)$ on $T^{2}$ such that, for $\alpha$-almost all $a^{1} \in A$,

$$
\bar{\Pi}\left(\tau\left(\cdot, a^{1}\right)^{-1}\left(B_{1}\right) \cap \tau\left(\cdot, a^{2}\right)^{-1}\left(B_{2}\right)\right)=p_{a}\left(B_{1} \times B_{2}\right)=p_{a}\left(B_{2} \times B_{1}\right)
$$

for $\alpha$-almost all $a^{2} \in A$ and all Borel sets $B_{1}, B_{2} \subset T$.

(c) If $\mathcal{D} \subset \bar{\Phi}$ is the Borel $\sigma$-algebra that is generated be the mapping $\varphi \rightarrow$ $D\left(\left\{\tau\left(\varphi, a^{\prime}\right)\right\}_{a^{\prime} \in A}\right)$, then conditionally on $\mathcal{D}$, the random variables $\tau\left(\cdot, a^{\prime}\right)$, $a^{\prime} \in A$, are essentially pairwise conditionally independent and identically distributed. Moreover, the sample distribution $D\left(\left\{\tau\left(\varphi, a^{\prime}\right)\right\}_{a^{\prime} \in A}\right)$ of types in the population is almost surely equal to the common conditonal distribution of types in the population, i..e., for $\alpha$-almost all $a \in A, D\left(\left\{\tau\left(\cdot, a^{\prime}\right)\right\}_{a^{\prime} \in A}\right)$ is a regular conditional distribution for $\tau(\cdot, a)$ given $\mathcal{D}$.

Proposition 5.1 provides a mathematical foundation for the appliedtheory work mentioned in the introduction where uncertainty is assumed to have an aggregate component and an agent-specific component, with symmetry and a law of large numbers holding for the latter. Whereas this decomposition of uncertainty is usually introduced ad hoc in order to simplify the analysis, Proposition 5.1 shows that it is actually implied by the

\footnotetext{
${ }^{15}$ Harsanyi (1967/68), Samet (1998 a, b), Feinberg (2000), Rodriguez-Neto (2010), Hellman and Samet (2012), Hellwig (2012).

${ }^{16}$ Hellman and Samet (2012).
} 
assumption of anonymity in beliefs. In settings in which agents do not care about the names of the other agents but only about the distribution of the other agents' types, uncertainty can always be decomposed into an aggregate component and an agent-specific component. The aggregate component concerns the cross-section distribution of types, the agent-specific component concerns the different agents' individual types. Conditionally on the aggregate component, the agent-specific components are essentially (pairwise) independent and identically distributed, the conditional distribution of any one agent's type is equal to the cross-section distribution, and an exact (conditional) law of large numbers holds.

\subsection{Common Priors and Macro Belief Functions}

Whereas the belief functions $b_{a}$ in Proposition 5.1 differ across agents, due to their having different domains and ranges, the exchangeability of the random variables $\tau\left(\cdot, a^{\prime}\right), a^{\prime} \in A$, ensures that the associated macro belief functions are the same for all agents. Thus, for any incomplete-information model that admits a common prior and has beliefs satisfying the condition of anonymity in beliefs, there exists a function $\beta: T \rightarrow \mathcal{M}(\mathcal{M}(T))$ such that, for any agent $a, \beta(\tau(\cdot, a))$ is a regular conditional distribution for $D\left(\left\{\tau\left(\cdot, a^{\prime}\right)\right\}_{a^{\prime} \in A}\right)$ given $\tau(\cdot, a)$. Moreover, of the belief functions $b_{a}, a \in A$, are coherent and measurable, $\beta$ is also coherent and measurable.

Suppose we take the macro belief function $\beta$ as given. If $\beta$ is coherent and measurable, can we infer the existence of a set of belief functions $b_{a}, a \in A$, satisfying anonymity in beliefs such that the incomplete-information model $\left\{T, \Theta, \theta_{a}, b_{a}\right\}_{a \in A}$ admits a common prior and $\beta$ is the associated macro belief function? The answer to this question turns out to be negative. This finding contrasts with Proposition 4.6 showing that for any coherent and measurable macro belief function $\beta$ and any $a \in A$, there exist an agent-specific prior and a coherent and measurable belief function $b_{a}$ satisfying anonymity in beliefs such that, under the given prior, $b_{a}\left(t_{a}\right)$ is a conditional probability distribution for $\left\{\tau\left(\cdot, a^{\prime}\right)\right\}_{a^{\prime} \in A}$ given that the agent's own type is $t_{a}$.

In the proof of Proposition 4.6, the random variable $\tau(\cdot, a)$ for the agent's own type was specified without regard to the other agents' beliefs; the marginal distribution $\Psi_{\beta}^{T}$ of this random variable was arbitrary. In a model with a common prior, $\tau(\cdot, a)$ cannot be specified arbitrarily but must be consistent with the other agents' beliefs. The following result gives a necessary and sufficient condition for this requirement to be satisfied.

Proposition 5.2 Let $(\Phi, \bar{\Phi}, \bar{\Pi})$ be a complete probability space and let $(\Phi \times$ 
$A, \bar{\Phi} \otimes \mathcal{A}, \bar{\Pi} \otimes \alpha)$ be a rich Fubini extension. For any coherent measurable function $\beta: T \rightarrow \mathcal{M}(\mathcal{M}(T))$, the following statements are equivalent.

(a) There exists a mapping $\tau: \Phi \times A \rightarrow T$ that is measurable with respect to $\bar{\Phi} \otimes \mathcal{A}$ and coherent and measurable belief functions $b_{a}(\cdot), a \in A$, such that, for $\alpha$-almost every $a \in A, b_{a}(\tau(\cdot, a))$ is a regular conditional distribution for $\left\{\tau\left(\cdot, a^{\prime}\right)\right\}_{a^{\prime} \in A_{-a}}$ given $\tau(\cdot, a)$, and, moreover, for all $t_{a} \in T, b_{a}\left(t_{a}\right)$ satisfies anonymity in beliefs and the associated macro belief $b_{a}^{*}\left(t_{a}\right)$ coincides with $\beta\left(t_{a}\right)$.

(b) There exist distributions $\Psi_{\beta}^{T}$ on $T$ and $\Psi_{\beta}^{\mathcal{M}(T)}$ on $\mathcal{M}(T)$ such that, for all measurable sets $B_{t} \subset T$ and $B_{\delta} \subset \mathcal{M}(T)$,

$$
\int_{B_{\delta}} \delta\left(B_{t}\right) d \Psi_{\beta}^{\mathcal{M}(T)}(\delta)=\int_{B_{t}} \beta\left(B_{\delta} \mid t\right) d \Psi_{\beta}^{T}(t)
$$

Proof. Suppose that statement (a) is true. Let $\Psi_{\beta}$ be the joint distribution of the pair $\left(\tau(\cdot, a), D\left(\left\{\tau\left(\cdot, a^{\prime}\right)\right\}_{a^{\prime} \in A_{-a}}\right)\right)$. Let $a$ be such that, for all $t_{a} \in T$, $b_{a}\left(t_{a}\right)$ satisfies anonymity in beliefs and the associated macro belief $b_{a}^{*}\left(t_{a}\right)$ satisfies $b_{a}^{*}\left(t_{a}\right)=\beta\left(t_{a}\right)$. If $\Psi_{\beta}^{T} \in \mathcal{M}(T)$ and $\Psi_{\beta}^{\mathcal{M}(T)} \in \mathcal{M}(\mathcal{M}(T))$ are the associated marginal distributions on $T$ and $\mathcal{M}(T)$, then, for all measurable sets $B_{t} \subset T$ and $B_{\delta} \subset \mathcal{M}(T)$,

$$
\Psi_{\beta}\left(B_{t} \times B_{\delta}\right)=\int_{B_{t}} \beta\left(B_{\delta} \mid t\right) d \Psi_{\beta}^{T}(t)
$$

and

$$
\Psi_{\beta}\left(B_{t} \times B_{\delta}\right)=\int_{B_{\delta}} \delta\left(B_{t}\right) d \Psi_{\beta}^{\mathcal{M}(T)}(\delta) .
$$

Equation (5.3) must hold because, by the definition of the macro belief function $b_{a}^{*}$ and the coincidence of $b_{a}^{*}$ and $\beta, \beta(\tau(\cdot, a))$ is a regular conditional distribution for $D\left(\left\{\tau\left(\cdot, a^{\prime}\right)\right\}_{a^{\prime} \in A_{-a}}\right)$ given $\tau(\cdot, a)$ and, moreover, by the atomlessness of $\alpha, D\left(\left\{\tau\left(\cdot, a^{\prime}\right)\right\}_{a^{\prime} \in A_{-a}}\right)=D\left(\left\{\tau\left(\cdot, a^{\prime}\right)\right\}_{a^{\prime} \in A}\right)$. Equation (5.4) must hold because, by the assumption that, for all $t_{a} \in T, b_{a}\left(t_{a}\right)$ satisfies anonymity in beliefs, statement (c) in Proposition 4.5 implies that $D\left(\left\{\tau\left(\cdot, a^{\prime}\right)\right\}_{a^{\prime} \in A_{-a}}\right)=D\left(\left\{\tau\left(\cdot, a^{\prime}\right)\right\}_{a^{\prime} \in A}\right)$ is a regular conditional distribution for $\tau(\cdot, a)$ given $D\left(\left\{\tau\left(\cdot, a^{\prime}\right)\right\}_{a^{\prime} \in A_{-a}}\right)$. Upon combining (5.3) and (5.4), one obtains (5.2), which proves statement (b).

Conversely, suppose that statement (b) is true. Fix some $a \in A$. Use the construction in the proof of Proposition 4.6 with the given $\Psi_{\beta}^{T}$ to obtain the desired $\tau(\cdot, \cdot)$ and $b_{a}(\cdot)$ (for this particular agent). The joint distribution of the pair $\left(\tau(\cdot, a), D\left(\left\{\tau\left(\cdot, a^{\prime}\right)\right\}_{a^{\prime} \in A_{-a}}\right)\right)$ is then given by (5.3). By Proposition 4.5, for $\alpha$-almost all $a^{\prime \prime} \in A_{-a}, D\left(\left\{\tau\left(\cdot, a^{\prime}\right)\right\}_{a^{\prime} \in A_{-a}}\right)$ is a regular 
conditional distribution for $\tau\left(\cdot, a^{\prime \prime}\right)$ given $D\left(\left\{\tau\left(\cdot, a^{\prime}\right)\right\}_{a^{\prime} \in A_{-a}}\right)$. By (5.4), it follows that, for $\alpha$-almost all $a^{\prime \prime} \in A_{-a}, \Psi_{\beta}$ is also the joint distribution of the pair $\left(\tau\left(\cdot, a^{\prime \prime}\right), D\left(\left\{\tau\left(\cdot, a^{\prime}\right)\right\}_{a^{\prime} \in A_{-a}}\right)\right)$. By another application of $(5.3)$, therefore, $\beta\left(\tau\left(\cdot, a^{\prime \prime}\right)\right)$ is a regular conditional distribution for $D\left(\left\{\tau\left(\cdot, a^{\prime}\right)\right\}_{a^{\prime} \in A}\right)=$ $D\left(\left\{\tau\left(\cdot, a^{\prime}\right)\right\}_{a^{\prime} \in A_{-a^{\prime \prime}}}\right)$ given $\tau\left(\cdot, a^{\prime \prime}\right)$. Upon setting

$$
\left.b_{a^{\prime \prime}}\left(t_{a^{\prime \prime}}\right)=\int_{\mathcal{M}(T)} \hat{b}_{a^{\prime \prime}}(\delta) d \beta\left(\delta \mid t_{a^{\prime \prime}}\right)\right),
$$

with $\hat{b}_{a^{\prime \prime}}$ given by Remark 3.7, one obtains the desired belief function $b_{a^{\prime \prime}}$ for agent $a^{\prime \prime}$. The same arguments as in the proof of Proposition 4.5 show that this belief function has the properties postulated in statement (a).

There are two ways to think about the joint distribution $\Psi_{\beta}$ of the pair $\left(\tau(\cdot, a), D\left(\left\{\tau\left(\cdot, a^{\prime}\right)\right\}_{a^{\prime} \in A_{-a}}\right)\right)$. One way is to think about $\tau(\cdot, a)$ as given and to derive $\Psi_{\beta}$ from the distribution $\Psi_{\beta}^{T}$ of $\tau(\cdot, a)$ and the macro belief function $\beta$. Another way is to think about $D\left(\left\{\tau\left(\cdot, a^{\prime}\right)\right\}_{a^{\prime} \in A_{-a}}\right)=D\left(\left\{\tau\left(\cdot, a^{\prime}\right)\right\}_{a^{\prime} \in A}\right)$ as

given and to derive $\Psi_{\beta}$ from the distribution $\Psi_{\beta}^{\mathcal{M}(T)}$ of $D\left(\left\{\tau\left(\cdot, a^{\prime}\right)\right\}_{a^{\prime} \in A}\right)$ and the fact that, conditionally on $D\left(\left\{\tau\left(\cdot, a^{\prime}\right)\right\}_{a^{\prime} \in A}\right), \tau(\cdot, a)$ is distributed as $D\left(\left\{\tau\left(\cdot, a^{\prime}\right)\right\}_{a^{\prime} \in A}\right)$. Statement (b) in Proposition 5.2 ensures that these two approaches are mutually consistent.

\subsection{Existence of a Common Prior}

The condition that Proposition 5.2 gives for the existence of a common prior in a model with a continuum of agents and anonymity in beliefs is formally equivalent to a condition for the existence of a common prior in a certain two-player model. In this two-player model, player 1 has the type space $T$ and player 2 the type space $\mathcal{M}(T)$. Beliefs are given by the functions $t \rightarrow \beta(t)$ and $\delta \rightarrow \delta$. For each $t \in T$, the probabilistic beliefs of player 1 about the type of player 2 are given by $\beta(t) \in \mathcal{M}(\mathcal{M}(T))$; for each $\delta \in \mathcal{M}(T)$, the probabilistic beliefs of player 2 about the type of player 1 are given by $\delta$. For given $\beta$, a measure $\Psi_{\beta} \in \mathcal{M}(T \times \mathcal{M}(T))$ that satisfies statement (b) in Proposition 5.2 exists if and if $\Psi_{\beta}$ is a common prior for the specified two-player model with belief functions $t \rightarrow \beta(t)$ and $\delta \rightarrow \delta$.

Given this equivalence, I use arguments from the analysis of two-player games to give a condition on the macro belief function $\beta$ under which this function is compatible with the existence of a common prior. I begin with a lemma showing that coherent macro beliefs have densities and that these densities are positive. 
Lemma 5.3 If the macro belief function $\beta(\cdot)$ is coherent, there exists a measurable functions $f: \mathcal{M}(T) \times T \times T \rightarrow \mathbb{R}_{+}$such that, for any $t$ and $t^{\prime}$ in $T$ and any measurable set $B_{\delta} \subset \mathcal{M}(T)$,

$$
\beta\left(B_{\delta} \mid t^{\prime}\right)=\int_{B_{\delta}} f\left(\delta, t^{\prime}, t\right) \beta(d \delta \mid t) .
$$

The function $f$ satisfies the equation

$$
f\left(\delta, t^{\prime \prime}, t\right)=f\left(\delta, t^{\prime \prime}, t^{\prime}\right) \cdot f\left(\delta, t^{\prime}, t\right)
$$

for $\beta(t)$-almost all $\delta \in \mathcal{M}(T)$, for all $t, t^{\prime}, t^{\prime \prime}$ in $T$. Moreover,

$$
f\left(\delta, t, t^{\prime}\right)>0
$$

for $\beta\left(t^{\prime}\right)$-almost all $\delta \in D$, for all $t, t^{\prime} \in T$.

Proof. The first statement of the lemma follows from strong coherence and the Radon-Nikodym Theorem. To prove the second statement, it suffices to note that, for any measurable set $B_{\delta} \subset \mathcal{M}(T)$ and any $t, t^{\prime}, t^{\prime \prime}$, the first statement implies

$$
\beta\left(B_{\delta} \mid t^{\prime \prime}\right)=\int_{B_{\delta}} f\left(\delta, t^{\prime \prime}, t^{\prime}\right) d \beta\left(\delta \mid t^{\prime}\right)=\int_{B_{\delta}} f\left(\delta, t^{\prime \prime}, t^{\prime}\right) \cdot f\left(\delta, t^{\prime}, t\right) d \beta(\delta \mid t),
$$

so that the product $f\left(\cdot, t^{\prime \prime}, t^{\prime}\right) \cdot f\left(\cdot, t^{\prime}, t\right)$ is a Radon-Nikodym derivative of the measure $\beta\left(t^{\prime \prime}\right)$ with respect to the measure $\beta(t)$. The statement follows because the Radon-Nikodym derivative of one measure with respect to another is unique up to a set of measure zero.

To prove the last statement of the lemma, fix $t^{\prime} \in T$ and $\delta^{\prime} \in D$. Upon setting $t^{\prime \prime}=t$ in (5.6), one finds that $f\left(\delta, t, t^{\prime}\right) \cdot f\left(\delta, t^{\prime}, t\right)=1$ for $\beta(t)$-almost all $\delta \in \mathcal{M}(T)$, for all $t$ and $t^{\prime}$ in $T$. Therefore $f\left(\delta, t, t^{\prime}\right)>0$ for $\beta(t)$-almost all $\delta \in \mathcal{M}(T)$, for all $t$ and $t^{\prime}$ in $T$. The last statement of the lemma follows because the measures $\beta(t)$ and $\beta\left(t^{\prime}\right)$ are mutually absolutely continuous.

I next introduce a property ensuring that cross-section type distributions in the support of the macro beliefs $\beta(t), t \in T$, also have positive densities. For lack of a better term, I call this property strong coherence.

Strong Coherence of Macro Belief Functions A macro belief function $\beta: T \rightarrow \mathcal{M}(\mathcal{M}(T))$ is said to be strongly coherent if it is coherent and if there exists a set $D \subset \mathcal{M}(T)$ such that $\beta(D \mid t)=1$ for all $t \in T$ and the measures $\delta \in D$ are mutually absolutely continuous. 
Lemma 5.4 If the macro belief function $\beta(\cdot)$ is strongly coherent, there exists a measurable function $g: T \times D \times D \rightarrow \mathbb{R}_{+}$such that, for any $\delta$ and $\delta^{\prime}$ in the specified set $D$ and any measurable set $B_{t} \subset T$,

$$
\delta^{\prime}\left(B_{t}\right)=\int_{B_{t}} g\left(t, \delta^{\prime}, \delta\right) \delta(d t)
$$

The function $g$ satisfies the equation

$$
g\left(t, \delta^{\prime \prime}, \delta\right)=g\left(t, \delta^{\prime \prime}, \delta^{\prime}\right) \cdot g\left(t, \delta^{\prime}, \delta\right)
$$

for $\delta$-almost all $t \in T$, for all $\delta, \delta^{\prime}, \delta^{\prime \prime}$ in D. Moreover,

$$
g\left(t, \delta, \delta^{\prime}\right)>0
$$

for $\delta^{\prime}$-almost all $t \in T$, for all $\delta$ all $\delta^{\prime} \in D$.

The proof of Lemma 5.4 is step for step the same as the proof of Lemma 5.3 and is left to the reader.

Proposition 5.5 A strongly coherent macro belief function $\beta: T \rightarrow \mathcal{M}(\mathcal{M}(T))$ admits a common prior if and only if the associated density functions $f$ and $g$ satisfy the equation

$$
\begin{aligned}
& g\left(t_{1}, \delta_{1}, \delta_{0}\right) \cdot f\left(\delta_{1}, t_{2}, t_{0}\right) \cdot g\left(t_{2}, \delta_{2}, \delta_{0}\right) \cdot f\left(\delta_{2}, t_{1}, t_{0}\right) \\
= & f\left(\delta_{1}, t_{1}, t_{0}\right) \cdot g\left(t_{1}, \delta_{2}, \delta_{0}\right) \cdot f\left(\delta_{2}, t_{2}, t_{0}\right) \cdot g\left(t_{2}, \delta_{1}, \delta_{0}\right)
\end{aligned}
$$

for any $t_{0} \in T$ and $\delta_{0} \in D$, for $\beta\left(t_{0}\right)$-almost all $\delta_{1}$ and $\delta_{2}$ in $D$ and $\delta_{0}$-almost all $t_{1}$ and $t_{2}$ in $T$.

Proof. If $\beta$ admits a common prior, then statement (b) in Proposition 5.2 holds. Given the marginal distributions $\Psi_{\beta}^{T}$ and $\Psi_{\beta}^{\mathcal{M}(T)}$ specified let there, let $\Psi_{\beta}$ be the distribution on $\mathcal{M}(T \times \mathcal{M}(T))$ that is given by (5.3) and (5.4).

I note that the measure $\Psi_{\beta}^{\mathcal{M}(T)}$ and any one of the measures $\beta\left(t_{0}\right), t_{0} \in T$, are mutually absolutely continuous: For $B_{t}=T$ and any $t_{0} \in T,(5.3)$ and Lemma 5.3 imply

$$
\begin{aligned}
\Psi_{\beta}^{\mathcal{M}(T)}\left(B_{\delta}\right) & =\int_{T} \beta\left(B_{\delta} \mid t\right) d \Psi_{\beta}^{T}(t) \\
& =\int_{T} \int_{B_{\delta}} f\left(\delta, t, t_{0}\right) d \beta\left(\delta \mid t_{0}\right) d \Psi_{\beta}^{T}(t) \\
& =\int_{B_{\delta}} \int_{T} f\left(\delta, t, t_{0}\right) d \Psi_{\beta}^{T}(t) d \beta\left(\delta \mid t_{0}\right)
\end{aligned}
$$


Absolute continuity of $\Psi_{\beta}^{\mathcal{M}(T)}$ with respect to $\beta\left(t_{0}\right)$ is immediate. Absolute continuity of $\beta\left(t_{0}\right)$ with respect to $\Psi_{\beta}^{\mathcal{M}(T)}$ follows from the strict positivity of $f\left(\delta, t, t_{0}\right)$.

I next claim that the measure $\Psi_{\beta}^{T}$ and any measure $\delta_{0} \in D$ are mutually absolutely continuous. To see this, note that, for $B_{\delta}=D$, equation (5.4) implies

$$
\begin{aligned}
\Psi_{\beta}^{T}\left(B_{t}\right) & =\int_{D} \delta\left(B_{t}\right) d \Psi_{\beta}^{\mathcal{M}(T)}(\delta) \\
& =\int_{D} \int_{B_{t}} g\left(t, \delta, \delta_{0}\right) d \delta_{0}(t) d \Psi_{\beta}^{\mathcal{M}(T)}(\delta) \\
& =\int_{B_{t}} \int_{D} g\left(t, \delta, \delta_{0}\right) d \Psi_{\beta}^{\mathcal{M}(T)}(\delta) d \delta_{0}(t)
\end{aligned}
$$

for any $\delta_{0} \in D$. Hence $\Psi_{\beta}^{T}$ is absolutely continuous with respect to $\delta_{0}$. By the strict positivity of $g\left(t, \delta, \delta_{0}\right),(5.13)$ also implies that $\delta_{0}$ is absolutely continuous with respect to $\Psi_{\beta}^{T}$.

Equations (5.13) and (5.12) also imply that the Radon-Nikodym derivatives of $\Psi_{\beta}^{T}$ and $\Psi_{\beta}^{\mathcal{M}(T)}$ with respect to $\delta_{0}$ and $\beta\left(t_{0}\right)$ are given as

$$
\psi_{t}\left(\cdot \mid \delta_{0}\right):=\int_{D} g(\cdot, \delta) d \Psi_{\beta}^{\mathcal{M}(T)}(\delta)
$$

and

$$
\psi_{\delta}\left(\cdot \mid t_{0}\right):=\int_{T} f\left(\cdot, t, t_{0}\right) d \Psi_{\beta}^{T}(t) .
$$

Thus, equations (5.3) and (5.4) can be rewritten in the form

$$
\Psi_{\beta}\left(B_{t} \times B_{\delta}\right)=\int_{B_{\delta}} \int_{B_{t}} g(t, \delta) \cdot \psi_{\delta}\left(\delta \mid t_{0}\right) d \delta_{0}(t) d \beta\left(\delta \mid t_{0}\right)
$$

and

$$
\Psi_{\beta}\left(B_{t} \times B_{\delta}\right)=\int_{B_{t}} \int_{B_{\delta}} f\left(\delta, t, t_{0}\right) \cdot \psi_{t}\left(t \mid \delta_{0}\right) d \beta\left(\delta \mid t_{0}\right) d \delta_{0}(t) .
$$

One easily sees that $\Psi_{\beta}$ is absolutely continuous with respect to the product measure $\beta\left(t_{0}\right) \times \delta_{0}$, with a density $\psi\left(\cdot, \cdot \mid t_{0}, \delta_{0}\right)$ satisfying

$$
\psi\left(t, \delta \mid t_{0}, \delta_{0}\right)=g\left(t, \delta, \delta_{0}\right) \cdot \psi_{\delta}\left(\delta \mid t_{0}\right)=f\left(\delta, t, t_{0}\right) \cdot \psi_{t}\left(t \mid \delta_{0}\right)
$$

for $\delta_{0}$-almost all $t \in T$ and $\beta\left(t_{0}\right)$-almost all $\delta \in D$. 
Consider the second equation in (5.16) with $t$ and $\delta$ replaced by different constellations of $t_{1}, t_{2} \in T$ and $\delta_{1}, \delta_{2} \in D$. This yields the equation

$$
\begin{gathered}
g\left(t_{1}, \delta_{1}\right) \cdot \psi_{\delta}\left(\delta_{1}, t_{0}\right) \cdot f\left(\delta_{1}, t_{2}, t_{0}\right) \cdot \psi_{t}\left(t_{2}\right) \\
\cdot g\left(t_{2}, \delta_{2}\right) \cdot \psi_{\delta}\left(\delta_{2}, t_{0}\right) \cdot f\left(\delta_{2}, t_{1}, t_{0}\right) \cdot \psi_{t}\left(t_{1}\right) \\
= \\
f\left(\delta_{1}, t_{1}, t_{0}\right) \cdot \psi_{t}\left(t_{1}\right) \cdot g\left(t_{1}, \delta_{2}\right) \cdot \psi_{\delta}\left(\delta_{2}, t_{0}\right) \\
\cdot f\left(\delta_{2}, t_{2}, t_{0}\right) \cdot \psi_{t}\left(t_{2}\right) \cdot g\left(t_{2}, \delta_{1}\right) \cdot \psi_{\delta}\left(\delta_{1}, t_{0}\right) .
\end{gathered}
$$

Because the measures $\Psi_{\beta}^{T}$ and $\delta_{0}$, as well as the measures $\Psi_{\beta}^{\mathcal{M}(T)}$ and $\beta\left(t_{0}\right)$, are mutually absolutely continuous, the same argument as in the proof of Lemma 5.3 implies that

$$
\psi_{t}\left(t, \delta_{0}\right)>0 \text { for } \delta_{0} \text {-almost all } t
$$

and

$$
\psi_{\delta}\left(\delta, t_{0}\right)>0 \text { for } \beta\left(t_{0}\right) \text {-almost all } \delta .
$$

For $\delta_{0}$-almost all $t_{1}$ and $t_{2}$ in $T$ and $\beta\left(t_{0}\right)$-almost all $\delta_{1}$ and $\delta_{2}$ in $D$, therefore, the terms $\psi_{\delta}\left(\delta_{1}, t_{0}\right), \psi_{t}\left(t_{2}\right), \psi_{\delta}\left(\delta_{2}, t_{0}\right)$, and $\psi_{t}\left(t_{1}\right)$ in (5.17) can be cancelled, which yields (5.11). Because the choice of $t_{0}$ and $\delta_{0}$ was arbitrary, the conclusion holds for all $t_{0}$.

To prove the converse, suppose that, for some $t_{0} \in T$ and $\delta_{0} \in D$, equation (5.11) is satisfied for $\delta_{0}$-almost all $t_{1}$ and $t_{2}$ in $T$ and $\beta\left(t_{0}\right)$-almost all $\delta_{1}$ and $\delta_{2}$ in $D$. By Lemma 5.3, there exist some $t_{1}$ and $\delta_{1}$ so that

$$
f\left(\delta_{1}, t_{1}, t_{0}\right)>0, \quad g\left(t_{1}, \delta_{1}\right)>0
$$

and

$$
g\left(t_{1}, \delta\right)>0 \text { for } \beta\left(t_{0}\right) \text {-almost all } \delta .
$$

For any $t$ and $\delta$, define

$$
\begin{gathered}
\lambda\left(t_{0}, \delta_{0}\right):=\left[\int_{D} \int_{T} \frac{f\left(\delta, t_{1}, t_{0}\right)}{f\left(\delta_{1}, t_{1}, t_{0}\right)} \cdot \frac{g\left(t, \delta, \delta_{0}\right)}{g\left(t_{1}, \delta, \delta_{0}\right)} d \delta_{0}(t) d \beta\left(\delta \mid t_{0}\right)\right]^{-1}, \\
\psi\left(t, \delta \mid t_{0}, \delta_{0}\right):=\lambda\left(t_{0}, \delta_{0}\right) \cdot \frac{f\left(\delta, t_{1}, t_{0}\right)}{f\left(\delta_{1}, t_{1}, t_{0}\right)} \cdot \frac{g\left(t, \delta, \delta_{0}\right)}{g\left(t_{1}, \delta, \delta_{0}\right)}
\end{gathered}
$$

if $g\left(t_{1}, \delta, \delta_{0}\right)>0$, and

$$
\psi\left(t, \delta \mid t_{0}, \delta_{0}\right)::=0
$$


if $g\left(t_{1}, \delta, \delta_{0}\right)=0$. Further define a measure $\Psi_{\beta} \in \mathcal{M}(T \times D)$ by setting

$$
\Psi_{\beta}\left(B_{t} \times B_{\delta}\right):=\int_{B_{\delta}} \int_{B_{t}} \psi\left(t, \delta \mid t_{0}, \delta_{0}\right) d \delta_{0}(t) d \beta\left(\delta \mid t_{0}\right)
$$

for any measurable $B_{t} \subset T$ and $B_{\delta} \subset D$. Then, by construction,

$$
\begin{aligned}
\Psi_{\beta}\left(B_{t} \times B_{\delta}\right) & =\int_{B_{\delta}} \int_{B_{t}} \lambda \cdot \frac{f\left(\delta, t_{1}, t_{0}\right)}{f\left(\delta_{1}, t_{1}, t_{0}\right)} \cdot \frac{g\left(t, \delta, \delta_{0}\right)}{g\left(t_{1}, \delta, \delta_{0}\right)} d \delta_{0}(t) d \beta\left(\delta \mid t_{0}\right) \\
& =\int_{B_{\delta}} \int_{B_{t}} g\left(t, \delta, \delta_{0}\right) d \delta_{0}(t) \cdot d \Psi_{\beta}^{\mathcal{M}(T)}(\delta) \\
& =\int_{B_{\delta}} \int_{B_{t}} d \delta(t) \cdot d \Psi_{\beta}^{\mathcal{M}(T)}(\delta)
\end{aligned}
$$

where $\Psi_{\beta}^{\mathcal{M}(T)} \in \mathcal{M}(\mathcal{M}(T))$ is given by the formula

$$
\Psi_{\beta}^{\mathcal{M}(T)}\left(B_{\delta}\right)=\Psi_{\beta}\left(T \times B_{\delta}\right)=\int_{B_{\delta}} \lambda \cdot \frac{f\left(\delta, t_{1}, t_{0}\right)}{f\left(\delta_{1}, t_{1}, t_{0}\right)} \cdot \frac{1}{g\left(t_{1}, \delta, \delta_{0}\right)} d \beta\left(\delta \mid t_{0}\right) .
$$

Equation (5.21) shows that $\Psi_{\beta}$ satisfies (5.4).

To verify that $\Psi_{\beta}$ also satisfies (5.3), observe that, by Lemma 5.3 and (5.19), we also have

$$
\psi\left(t, \delta \mid t_{0}, \delta_{0}\right)=\lambda \cdot \frac{f\left(\delta, t, t_{0}\right)}{f\left(\delta_{1}, t, t_{0}\right)} \cdot \frac{g\left(t, \delta_{1}, \delta_{0}\right)}{g\left(t_{1}, \delta_{1}, \delta_{0}\right)}
$$

for $\delta_{0} \times \beta\left(t_{0}\right)$-almost all $(t, \delta)$. Therefore, $(5.20)$ can be rewritten as

$$
\begin{aligned}
\Psi_{\beta}\left(B_{t} \times B_{\delta}\right) & =\int_{B_{t}} \int_{B_{\delta}} \lambda \cdot \frac{f\left(\delta, t, t_{0}\right)}{f\left(\delta_{1}, t, t_{0}\right)} \cdot \frac{g\left(t, \delta_{1}, \delta_{0}\right)}{g\left(t_{1}, \delta_{1}, \delta_{0}\right)} d \delta_{0}(t) d \beta\left(\delta \mid t_{0}\right) \\
& =\int_{B_{t}} \int_{B_{\delta}} f\left(\delta, t, t_{0}\right) d \beta\left(\delta \mid t_{0}\right) \cdot d \Psi_{\beta}^{T}(t) \\
& =\int_{B_{t}} \int_{B_{\delta}} d \beta(\delta \mid t) d \Psi_{\beta}^{T}(t)
\end{aligned}
$$

where $\Psi_{\beta}^{T} \in \mathcal{M}(T)$ is given by the formula

$$
\Psi_{\beta}^{T}\left(B_{t}\right)=\Psi_{\beta}\left(B_{t} \times D\right)=\int_{B_{t}} \lambda \cdot \frac{1}{f\left(\delta_{1}, t, t_{0}\right)} \cdot \frac{g\left(t, \delta_{1}, \delta_{0}\right)}{g\left(t_{1}, \delta_{1}, \delta_{0}\right)} d \delta_{0}(t) .
$$

Equation (5.23) shows that $\Psi_{\beta}$ also satisfies (5.3). Thus, the macro belief function $\beta$ satisfies statement (b) in Proposition 5.2. 
The consistency condition (5.11) is well known from Harsanyi (1967/68). Whereas most of the literature discusses this condition in terms of a twoplayer game with finitely many states, Proposition 4.5 shows that it also matters for the existence of a common prior in a large eocnomy with anonymity, with a potentially uncountable set of states. The underlying logic is the same: If distribution $\Psi_{\beta}$ has a density $\psi\left(\cdot, \cdot \mid t_{0}, \delta_{0}\right)$ with respect to the measure $\delta_{0} \times \beta\left(t_{0}\right)$, the ratio $\frac{\psi\left(t^{\prime}, \delta^{\prime} \mid t_{0}, \delta_{0}\right)}{\psi\left(t, \delta \mid t_{0}, \delta_{0}\right)}$ can be computed from the equation

$$
\frac{\psi\left(t^{\prime}, \delta^{\prime} \mid t_{0}, \delta_{0}\right)}{\psi\left(t, \delta \mid t_{0}, \delta_{0}\right)}=\frac{\psi\left(t^{\prime}, \delta \mid t_{0}, \delta_{0}\right)}{\psi\left(t, \delta \mid t_{0}, \delta_{0}\right)} \cdot \frac{\psi\left(t^{\prime}, \delta^{\prime} \mid t_{0}, \delta_{0}\right)}{\psi\left(t^{\prime}, \delta \mid t_{0}, \delta_{0}\right)}
$$

or from the equation

$$
\frac{\psi\left(t^{\prime}, \delta^{\prime} \mid t_{0}, \delta_{0}\right)}{\psi\left(t, \delta \mid t_{0}, \delta_{0}\right)}=\frac{\psi\left(t, \delta^{\prime} \mid t_{0}, \delta_{0}\right)}{\psi\left(t, \delta \mid t_{0}, \delta_{0}\right)} \cdot \frac{\psi\left(t^{\prime}, \delta^{\prime} \mid t_{0}, \delta_{0}\right)}{\psi\left(t, \delta^{\prime} \mid t_{0}, \delta_{0}\right)},
$$

and each time the result must be the same. If $\Psi_{\beta}$ satisfies (5.3) and (5.4), the density $\psi\left(\cdot, \cdot \mid t_{0}, \delta_{0}\right)$ satisfies (5.16), which yields

$$
\frac{\psi\left(t^{\prime}, \delta \mid t_{0}, \delta_{0}\right)}{\psi\left(t, \delta \mid t_{0}, \delta_{0}\right)}=\frac{g\left(t^{\prime}, \delta, \delta_{0}\right)}{g\left(t, \delta, \delta_{0}\right)}
$$

and

$$
\frac{\psi\left(t, \delta^{\prime} \mid t_{0}, \delta_{0}\right)}{\psi\left(t, \delta \mid t_{0}, \delta_{0}\right)}=\frac{f\left(\delta^{\prime}, t, t_{0}\right)}{f\left(\delta, t, t_{0}\right)}
$$

for any $t, t^{\prime} \in T$ and any $\delta, \delta^{\prime} \in D$ such that $\psi\left(t, \delta \mid t_{0}, \delta_{0}\right)>0$ (and therefore $g\left(t, \delta, \delta_{0}\right)>0$ and $\left.f\left(\delta, t, t_{0}\right)>0\right)$. When applied to (5.24), (5.26) and (5.27) yield

$$
\frac{\psi\left(t^{\prime}, \delta^{\prime} \mid t_{0}, \delta_{0}\right)}{\psi\left(t, \delta \mid t_{0}, \delta_{0}\right)}=\frac{f\left(\delta^{\prime}, t, t_{0}\right)}{f\left(\delta, t, t_{0}\right)} \cdot \frac{g\left(t^{\prime}, \delta^{\prime}, \delta_{0}\right)}{g\left(t, \delta^{\prime}, \delta_{0}\right)},
$$

when applied to (5.25),

$$
\frac{\psi\left(t^{\prime}, \delta^{\prime} \mid t_{0}, \delta_{0}\right)}{\psi\left(t, \delta \mid t_{0}, \delta_{0}\right)}=\frac{g\left(t^{\prime}, \delta, \delta_{0}\right)}{g\left(t, \delta, \delta_{0}\right)} \cdot \frac{f\left(\delta^{\prime}, t^{\prime}, t_{0}\right)}{f\left(\delta, t^{\prime}, t_{0}\right)} .
$$

For these two expressions to be compatible, one needs equation (5.11).

Notice that, according to (5.28) and (5.29), the ratio $\frac{\psi\left(t^{\prime}, \delta^{\prime} \mid t_{0}, \delta_{0}\right)}{\psi\left(t, \delta^{\prime} \mid t_{0}, \delta_{0}\right)}$ is uniquely determined by the density functions $f$ and $g$. Because the integral of $\psi\left(\cdot, \cdot \mid t_{0}, \delta_{0}\right)$ with respect to the measure $\delta_{0} \times \beta\left(t_{0}\right)$ must be equal to one, it follows that, up to modifications on a set of $\beta\left(t_{0}\right) \times \delta_{0}$-measure zero, the density function $\psi\left(\cdot, \cdot \mid t_{0}, \delta_{0}\right)$ and the measure $\Psi_{\beta}$ are uniquely determined by the functions $f$ and $g$. 
Remark 5.6 The common prior that is obtained if the condition in Proposition 5.2 holds is unique.

Remark 5.6 implies, in particular, that $\Psi_{\beta}$ does not depend on the pair $\left(t_{1}, \delta_{1}\right)$ that serves as a starting point for the construction in (5.18) - (5.20). The following remark shows that $\Psi_{\beta}$ also does not depend on the choice of the pair $\left(t_{0}, \delta_{0}\right)$ in equation (5.11) and the construction in (5.18) - (5.20).

Remark 5.7 The common prior that is obtained if the condition in Proposition 5.2 holds does not depend on the particular pair $\left(t_{0}, \delta_{0}\right)$ that are used in the construction.

Proof. I first note that the validity of (5.11) does not depend on the chosen pair $\left(t_{0}, \delta_{0}\right)$. To see this, multiply both sides of $(5.11)$ by the product $g\left(t_{1}, \delta_{0}, \delta_{0}^{\prime}\right) \cdot f\left(\delta_{1}, t_{0}, t_{0}^{\prime}\right) \cdot g\left(t_{2}, \delta_{0}, \delta_{0}^{\prime}\right) \cdot f\left(\delta_{2}, t_{0}, t_{0}^{\prime}\right)$, for some $t_{0}^{\prime} \in T$ and $\delta_{0}^{\prime} \in D$, and simplify the resulting equation using (5.6) and (5.9). The result is a version of $(5.11)$ with $\left(t_{0}, \delta_{0}\right)$ replaced by $\left(t_{0}^{\prime}, \delta_{0}^{\prime}\right)$.

To prove that $\Psi_{\beta}$ is unchanged if, in the construction (5.18) - (5.20), $\left(t_{0}, \delta_{0}\right)$ replaced by $\left(t_{0}^{\prime}, \delta_{0}^{\prime}\right)$, I note that, by Lemmas 5.3 and 5.4 , the righthand side of (5.20) can be written as

$$
\left.\int_{B_{\delta}} \int_{B_{t}} \frac{f\left(\delta, t_{1}, t_{0}\right) \cdot f\left(\delta, t_{0}, t_{0}^{\prime}\right)}{f\left(\delta_{1}, t_{1}, t_{0}\right)} \cdot \frac{g\left(t, \delta, \delta_{0}\right) \cdot g\left(t, \delta_{0}, \delta_{0}^{\prime}\right)}{g\left(t_{1}, \delta, \delta_{0}\right)} d \delta_{0}^{\prime}(t) d \beta\left(\delta \mid t_{0}^{\prime}\right)\right) .
$$

By another application of Lemmas 5.3 and 5.4, therefore, (5.20) can be rewritten as

$$
\begin{aligned}
\Phi\left(B_{t} \times B_{\delta}\right) & \left.=\int_{B_{\delta}} \int_{B_{t}} \lambda^{\prime} \cdot \frac{f\left(\delta, t_{1}, t_{0}^{\prime}\right)}{f\left(\delta_{1}, t_{1}, t_{0}^{\prime}\right)} \cdot \frac{g\left(t, \delta, \delta_{0}^{\prime}\right)}{g\left(t_{1}, \delta, \delta_{0}^{\prime}\right)} d \delta_{0}^{\prime}(t) d \beta\left(\delta \mid t_{0}^{\prime}\right)\right) \\
& \left.=\frac{\lambda^{\prime}}{\lambda\left(t_{0}^{\prime}, \delta_{0}^{\prime}\right)} \int_{B_{\delta}} \int_{B_{t}} \psi\left(t, \delta \mid t_{0}^{\prime}, \delta_{0}^{\prime}\right) d \delta_{0}^{\prime}(t) d \beta\left(\delta \mid t_{0}^{\prime}\right)\right)
\end{aligned}
$$

where $\lambda^{\prime}:=\lambda\left(t_{0}, \delta_{0}\right) \cdot f\left(\delta_{1}, t_{0}, t_{0}^{\prime}\right) \cdot g\left(t_{1}, \delta_{0}, \delta_{0}^{\prime}\right)$ and $\lambda\left(t_{0}^{\prime}, \delta_{0}^{\prime}\right)$ and $\psi\left(t, \delta \mid t_{0}^{\prime}, \delta_{0}^{\prime}\right)$ are given by $(5.18)$ and $(5.19)$ with $\left(t_{0}, \delta_{0}\right)$ replaced by $\left(t_{0}^{\prime}, \delta_{0}^{\prime}\right)$. For $B_{t}=T$ and $B_{\delta}=D$, we have $\Phi\left(B_{t} \times B_{\delta}\right)=1$ and $\left.\int_{B_{\delta}} \int_{B_{t}} \psi\left(t, \delta, t_{0}^{\prime}, \delta_{0}^{\prime}\right) \delta_{0}^{\prime}(d t) \beta\left(d \delta \mid t_{0}^{\prime}\right)\right)=$ 1. Therefore, $\lambda^{\prime}=\lambda\left(t_{0}^{\prime}, \delta_{0}^{\prime}\right)$, and (5.30) can be rewritten as

$$
\left.\Phi\left(B_{t} \times B_{\delta}\right)=\int_{B_{\delta}} \int_{B_{t}} \psi\left(t, \delta \mid t_{0}^{\prime}, \delta_{0}^{\prime}\right) \delta_{0}^{\prime}(d t) \beta\left(d \delta \mid t_{0}^{\prime}\right)\right)
$$

for all $B_{t}$ and $B_{\delta}$, which is just $(5.20)$ with $\left(t_{0}, \delta_{0}\right)$ replaced by $\left(t_{0}^{\prime}, \delta_{0}^{\prime}\right)$. 
Whereas the Harsanyi consistency condition is usually discussed as a necessary condition for the existence of a common prior, Proposition 4.5 shows that, under the given conditions, it is also sufficient. This result hinges on the strict positivity of the densities $f$ and $g$, which in turn is derived from the assumption that the macro belief function $\beta$ is strongly coherent. ${ }^{17}$

Strong coherence involves two properties, (i) coherence, i.e., mutual absolute continuity of the measures $\beta(t), t \in T$, and (ii) mutual absolute continuity of the measures $\delta \in D$, where $\beta(D \mid t)=1$ for all $t$. Coherence of the measures $\beta(t), t \in T$, is restrictive, but, as discussed in Section 4 , this property plays a key role in establishing that a given belief function $b_{a}$ admits even an agent-specific prior, let alone a common prior.

Given the coherence of the measures $\beta(t), t \in T$, the additional condition of mutual absolute continuity of the measures $\delta \in D$, where $\beta(D \mid t)=1$ for all $t$, is close to being necessary for the existence of a common prior (as well as being sufficient, in combination with the Harsanyi consistency condition).

Remark 5.8 Suppose that a coherent macro belief function $\beta: T \rightarrow \mathcal{M}(\mathcal{M}(T))$ admits a common prior $\Psi_{\beta} \in \mathcal{M}(T \times \mathcal{M}(T))$, with marginal distributions $\Psi_{\beta}^{T}, \Psi_{\beta}^{\mathcal{M}(T)}$ on $T$ and $\mathcal{M}(T)$. Then for every measurable set $B_{t} \subset T, \Psi_{\beta}^{T}\left(B_{t}\right)=$ 0 if and only if $\delta\left(B_{t}\right)=0$ for $\beta\left(t_{0}\right)$-almost all $\delta \in \mathcal{M}(T)$, for every $t_{0} \in T$.

Proof. Fix $B_{t} \subset T$. By (5.4),

$$
\Psi_{\beta}^{T}\left(B_{t}\right)=\int_{\mathcal{M}(T)} \delta\left(B_{t}\right) d \Psi_{\beta}^{\mathcal{M}(T)}(\delta)
$$

so $\Psi_{\beta}^{T}\left(B_{t}\right)=0$ implies $\Psi_{\beta}^{\mathcal{M}(T)}\left(B_{\delta}\left(B_{t}\right)\right)=1$, where

$$
B_{\delta}\left(B_{t}\right)=\left\{\delta \in \mathcal{M}(T) \mid \delta\left(B_{t}\right)=0\right\} .
$$

By (5.3) and Lemma 5.3, we also have

$$
\Psi_{\beta}^{\mathcal{M}(T)}\left(B_{\delta}\right)=\int_{B_{\delta}} \int_{T} f\left(\delta, t, t_{0}\right) d \Psi_{\beta}^{T}(t) d \beta\left(\delta \mid t_{0}\right)
$$

\footnotetext{
${ }^{17}$ This sufficiency result parallels the finding in Hellwig (2013) that, for games with finitely many players, if any element of any player's information partition intersects any element of any other player's information partition, then, for strictly positive belief systems, a common prior exists if the Harsanyi condition holds for all "cycles" of length four or less.
} 
for every measurable set $B_{\delta} \subset \mathcal{M}(T)$ and every $t_{0} \in T$. Since $f\left(\delta, t, t_{0}\right)$ is almost everywhere strictly positive, it follows that $\Psi_{\beta}^{\mathcal{M}(T)}$ and $\beta\left(t_{0}\right)$ are mutually absolutely continuous, so $\Psi_{\beta}^{\mathcal{M}(T)}\left(B_{\delta}\left(B_{t}\right)\right)=1$, i.e., $\Psi_{\beta}^{\mathcal{M}(T)}\left(\mathcal{M}(T) \backslash B_{\delta}\left(B_{t}\right)\right)=$ 0 , if and only if $\beta\left(\mathcal{M}(T) \backslash B_{\delta}\left(B_{t}\right)\right)=0$, i.e., $\beta\left(B_{\delta}\left(B_{t}\right) \mid t_{0}\right)=1$. The remark follows immediately.

In the condition that, for every $B_{t} \subset T, \Psi_{\beta}^{T}\left(B_{t}\right)=0$ if and only if $\delta\left(B_{t}\right)=0$ for $\beta\left(t_{0}\right)$-almost all $\delta \in \mathcal{M}(T)$, for every $t_{0} \in T$, the $\beta\left(t_{0}\right)$-null set of measures $\delta$ for which $\delta\left(B_{t}\right)>0$ even as $\Psi_{\beta}^{T}\left(B_{t}\right)=0$ might depend on $B_{t}$. Therefore this condition is slightly weaker than mutual absolute continuity of $\Psi_{\beta}^{T}$ and $\delta$, for $\beta\left(t_{0}\right)$-almost all $\delta \in \mathcal{M}(T)$, which in turn would imply strong coherence.

If $T$ was a finite set or if the measures $\beta(t), t \in T$, had finite supports, the condition given in Remark 5.8 would actually be sufficient for mutual absolute continuity of $\Psi_{\beta}^{T}$ and $\delta$, for $\beta\left(t_{0}\right)$-almost all $\delta \in \mathcal{M}(T)$, and therefore for strong coherence of $\beta$. In this case, Proposition 4.5 could be reformulated as a result about coherent macro belief functions, with strong coherence appearing as a part of the necessary and sufficient condition for the existence of a common prior. 


\section{References}

[1] Alesina, A., and G. Tabellini (1990), A Positive Theory of Fiscal Deficits and Government Debt, Review of Economic Studies 87, 403-414.

[2] Angeletos, G.-M., and I. Werning (2006), Crises and Prices: Information Aggregation, Multiplicity, and Volatility, American Economic Review 96 (5), 1720-1736.

[3] Bierbrauer, F.J., and M.F. Hellwig (2015), Public-Good Provision in Large Economies, Preprint 2015-12, Max Planck Institute for Research on Collective Goods, Bonn, http://www.coll.mpg.de/pdf_dat/2015_12online.pdf.

[4] Bryant, J. (1980), A Model of Reserves, Bank Runs, and Deposit Insurance, Journal of Banking and Finance 4, 335-344.

[5] Diamond, D.W., and P.H. Dybvig (1983), Bank Runs, Deposit Insurance, and Liquidity, Journal of Political Economy 91, 401-419.

[6] Feinberg, Y. (2000), Characterizing common priors in the form of posteriors, Journal of Economic Theory 91, 127-179.

[7] Feldman, M., and C. Gilles (1985), An expository note on individual risk without aggregate uncertainty, Journal of Economic Theory 35, $26-32$.

[8] Goldstein, I., and A. Pauzner (2005), Demand Deposit Contracts and the Probability of a Bank Run, Journal of Finance 60 (3), 1293-1327.

[9] Grossman, S.J., and O.D. Hart (1980), Takeover Bids, the Free-Rider Problem, and the Theory of the Corporation, Bell Journal of Economics, 11(1), 42-64.

[10] Gul, F., H. Sonnenschein, and R. Wilson (1986) Foundations of Dynamic Monopoly and the Coase Conjecture, Journal of Economic Theory $39,155-190$

[11] Hammond, P.J., and Y. Sun (2003), Monte Carlo Simulation of Macroeconomic Risk with a Continuum of Agents: the Symmetric Case, Economic Theory 21, 743-766

[12] Hammond, P.J., and Y. Sun (2006), The Essential Equivalence of Pairwise and Mutual Conditional Independence, Probability Theory and Related Fields 135, 415-427 
[13] Hammond, P.J., and Y. Sun (2008), Monte Carlo Simulation of Macroeconomic Risk with a Continuum of Agents: the General Case, Economic Theory 36, 303-325.

[14] Harsanyi, J.C. (1967/68), Games with Incomplete Information Played by Bayesian Players I, II, III, Management Science 14, 159 - 182; 320 334; 486-502.

[15] Hellman, Z., and D. Samet (2012), How Common are Common Priors?, Games and Economic Behavior 74, 517-525.

[16] Hellwig, C. (2002), Public Information, Private Information, and the Multiplicity of Equilibria in Coordination Games, Journal of Economic Theory 107(2), 191-222.

[17] Hellwig, M.F. (2013), From Posteriors to Priors via Cycles: An Addendum, Economics Letters 118, 455-458.

[18] Hellwig, M.F. (2011), Incomplete-Information Models of Large Economies with Anonymity: Existence and Uniqueness of Common Priors, Preprint, No. 2011/8, Max Planck Institute for Research on Collective Goods, Bonn. http://www.coll.mpg.de/pdf_dat/2011_08online.pdf.

[19] Judd, K.L. (1985), The law of large numbers with a continuum of IID random variables, Journal of Economic Theory 35, 19-25.

[20] Khan, M.A., and Y. Sun (1999), Non-cooperative games on hyperfinite Loeb spaces, Journal of Mathematical Economics 31, 455-492.

[21] Kingman, J.F.C. (1978), Uses of Exchangeability, Annals of Probability 6(2), 183-197.

[22] Kyle, A.S. (1985), Continuous Auctions and Insider Trading, Econometrica 53(6), 1315-1336.

[23] Kyle, A.S. (1989), Informed Speculation with Imperfect Competition, Review of Economic Studies 56 (3), Pages 317-355.

[24] Lindbeck, A., and J. Weibull (1987), Balanced-Budget Redistribution as the Outcome of Political Competition, Public Choice 52(3), 273-297.

[25] Mertens, J.-F., and S. Zamir (1985), Formulation of Bayesian analysis for games with incomplete information, International Journal of Game Theory 14, 1-29. 
[26] Morris, S. (1994), Trade with Heterogeneous Prior Beliefs and Asymmetric Information, Econometrica 62, 1326-1347.

[27] Morris, S., and H.S. Shin (1998), Unique Equilibrium in a Model of SelfFulfilling Currency Attacks, American Economic Review 88(3), 587597.

[28] Parthasarathy, K.R. (1967), Probability Measures on Metric Spaces, Academic Press, New York.

[29] Podczeck, K. (2010), On Existence of Rich Fubini Extensions, Economic Theory 45, 1-22.

[30] Qiao, L., Y. Sun, and Z. Zhang (2016), Conditional Exact Law of Large Numbers and Asymmetric Information Economies with Aggregate Uncertainty, Economic Theory 62(1-2), 43-64.

[31] Rochet, J.-C., and X. Vives (2004), Coordination Failures and the Lender of the Last Resort, Journal of the European Economic Association 2(6), 1116 - 1147.

[32] Rodrigues-Neto, J.A. (2009), From Posteriors to Priors via Cycles, Journal of Economic Theory 144, 876-883.

[33] Samet, D. (1998a), Iterative Expectations and Common Priors, Games and Economic Behavior 24, 131-141.

[34] Samet, D. (1998b), Common Priors and the Separation of Convex Sets, Games and Economic Behavior 24, 172-174.

[35] Sun, Y. (2006), The Exact Law of Large Numbers via Fubini Extension and Characterization of Insurable Risks, Journal of Economic Theory $126,31-69$.

[36] Sun, Y., and Y.Zhang (2009), Individual Risk and Lebesgue Extension without Aggregate Uncertainty, Journal of Economic Theory 144, 432442 . 